\title{
Boundary treatment for fourth-order staggered mesh discretizations of the incompressible Navier-Stokes equations
}

\author{
B. Sanderse ${ }^{\mathrm{a}, \mathrm{b}, *}$, R.W.C.P. Verstappen ${ }^{\mathrm{c}}$, B. Koren ${ }^{\mathrm{b}, \mathrm{d}}$ \\ a Energy Research Centre of the Netherlands (ECN), The Netherlands \\ b Centrum Wiskunde E' Informatica (CWI), The Netherlands \\ ${ }^{\mathrm{c}}$ Institute for Mathematics and Computing Science, University of Groningen, The Netherlands \\ d Department of Mathematics and Computer Science, Eindhoven University of Technology, The Netherlands
}

\section{A R T I C L E I N F O}

\section{Article history:}

Received 18 October 2012

Received in revised form 8 September 2013

Accepted 3 October 2013

Available online 10 October 2013

\section{Keywords:}

Incompressible Navier-Stokes equations

Symmetry preservation

Energy conservation

Boundary conditions

Fourth order accuracy

\begin{abstract}
A B S T R A C T
Harlow and Welch [Phys. Fluids 8 (1965) 2182-2189] introduced a discretization method for the incompressible Navier-Stokes equations conserving the secondary quantities kinetic energy and vorticity, besides the primary quantities mass and momentum. This method was extended to fourth order accuracy by several researchers $[25,14,21]$. In this paper we propose a new consistent boundary treatment for this method, which is such that continuous integration-by-parts identities (including boundary contributions) are mimicked in a discrete sense. In this way kinetic energy is exactly conserved even in case of nonzero tangential boundary conditions. We show that requiring energy conservation at the boundary conflicts with order of accuracy conditions, and that the global accuracy of the fourth order method is limited to second order in the presence of boundaries. We indicate how non-uniform grids can be employed to obtain full fourth order accuracy.
\end{abstract}

(c) 2013 Elsevier Inc. All rights reserved.

\section{Introduction}

Recently, symmetry-preserving and energy-conserving discretization methods have gained popularity for the solution of the incompressible Navier-Stokes equations $[26,18]$. In a symmetry-preserving discretization, the discrete difference operators mimic certain symmetry properties of the continuous differential operators. These discrete symmetry properties ensure that not only mass and momentum are conserved, but also derived quantities such as kinetic energy. Conservation of kinetic energy implies unconditional (non-linear) stability and absence of numerical diffusion. The latter is a favorable property when simulating turbulent flows with LES and DNS [9,13,17].

When combining symmetry preservation with high-order accuracy one can obtain very efficient methods for simulating turbulence [26]. Examples of high-order symmetry-preserving methods on Cartesian grids are the following. On uniform grids, Morinishi et al. [14] constructed a fourth order finite difference method which conserves mass, momentum and kinetic energy. On non-uniform grids strict conservation and a fourth order local truncation error could not be obtained. Vasilyev [21] uses the idea of symmetry preservation to extend the finite difference method of Morinishi et al. [10] to non-uniform grids, but he is not able to obtain simultaneous conservation of mass, momentum and energy on non-uniform grids (not even for the classic second-order method of Harlow and Welch). Ham et al. [5] show how the interpolation of the convective velocities should be performed on non-uniform meshes. They argue that second order is recovered for smoothly varying meshes. Verstappen and Veldman $[25,26]$ recognized that on non-uniform grids conservation could be obtained by respecting the symmetries of the underlying operators and keeping the weights of the difference operators mesh

\footnotetext{
* Corresponding author at: Energy research Centre of the Netherlands (ECN), The Netherlands.

E-mail addresses: bsanderse@gmail.com (B. Sanderse), r.w.c.p.verstappen@rug.nl (R.W.C.P. Verstappen), b.koren@tue.nl (B. Koren).
} 
independent. Their method (which is the finite-volume equivalent of [14] on uniform grids) conserves mass, momentum and kinetic energy on both uniform and non-uniform grids. Their results show that the local truncation error, on which [14,21,5] focus, does not have to be fourth order to let the global error be fourth order. This result is in agreement with theoretical work of Manteuffel and White [10].

An ongoing challenge in combining symmetry properties with high order is the prescription of discrete boundary conditions. Morinishi et al. [14] introduce ghost cells with values obtained by higher-order extrapolation and enforcing momentum conservation, but they do not respect the skew-symmetry of the convective operator. Furthermore pressure boundary conditions are prescribed, something which is not necessary in the original method of Harlow and Welch. Desjardins et al. [3] extend the approach of [14] to higher order. Their method is not energy-conserving for orders higher than two; the higher the order of the method, the larger the energy-conservation error. Again, explicit boundary conditions for the pressure are prescribed. The boundary conditions given in Verstappen and Veldman [26] are energy conserving for homogeneous Dirichlet conditions, but do not mimic the continuous integral properties in a discrete sense if the boundary conditions are non-homogeneous. Furthermore these conditions effectively introduce a Dirichlet condition for the pressure.

In this paper we propose new boundary conditions for the fourth order method such that mass, momentum and kinetic energy are conserved on a discrete level, including the case of non-homogeneous boundary conditions. First, in Section 2 we derive the conservation properties of the continuous equations from a number of integral identities. In Section 3 these integral identities are enforced in a discrete setting, for both the second and fourth order scheme, by a proper choice of boundary volumes and boundary conditions. In Section 4 we investigate the effect of the boundary treatment on the local and global error by employing a one-dimensional error analysis. This theoretical accuracy analysis is supported by numerical experiments in Section 5. In this section we also show the conservation properties of the newly proposed boundary conditions.

\section{Conservation properties of the continuous equations}

In this section we will discuss a number of properties of the continuous incompressible Navier-Stokes equations: conservation of mass, momentum and kinetic energy, and the equation for the pressure. These properties will be mimicked in a discrete sense in Section 3.

\subsection{Mass and momentum}

We consider the incompressible Navier-Stokes equations, written as

$$
\begin{aligned}
& \nabla \cdot \boldsymbol{u}=0, \\
& \frac{\partial \boldsymbol{u}}{\partial t}+\nabla \cdot(\boldsymbol{u} \boldsymbol{u})=-\nabla p+v \nabla^{2} \boldsymbol{u},
\end{aligned}
$$

in a domain $\Omega$ with boundary $\Gamma=\partial \Omega$, and supplemented with either no-slip boundary conditions

$$
\boldsymbol{u}=\boldsymbol{u}_{b} \text { on } \Gamma
$$

or periodic boundary conditions, and a divergence-free initial condition

$$
\boldsymbol{u}=\boldsymbol{u}_{0}
$$

See for example Gresho and Sani [4] for well-posedness of these equations. In integral form these equations read:

$$
\begin{aligned}
& \int_{\partial \Omega} \boldsymbol{u} \cdot \boldsymbol{n} \mathrm{d} \Gamma=0 \\
& \int_{\Omega} \frac{\partial \boldsymbol{u}}{\partial t} \mathrm{~d} \Omega+\int_{\partial \Omega} \boldsymbol{u} \boldsymbol{u} \cdot \boldsymbol{n} \mathrm{d} \Gamma=-\int_{\partial \Omega} p \boldsymbol{n} \mathrm{d} \Gamma+\int_{\partial \Omega} v \nabla \boldsymbol{u} \cdot \boldsymbol{n} \mathrm{d} \Gamma,
\end{aligned}
$$

expressing conservation of mass and momentum in the domain $\Omega$. In case of periodic boundary conditions, all boundary integrals disappear and one obtains the global momentum balance

$$
\frac{\mathrm{d}}{\mathrm{d} t} \int_{\Omega} \boldsymbol{u} \mathrm{d} \Omega=0 .
$$

In case the normal velocity vanishes at the boundary (no penetration, $\boldsymbol{u} \cdot \boldsymbol{n}=0$ ), only the contribution of the convective terms disappears. 


\subsection{Kinetic energy}

In incompressible flows the (kinetic) energy is a secondary conserved quantity, which follows from conservation of mass and momentum. The energy equation in integral form is derived by taking the inner product of the momentum equation with a function $\boldsymbol{v}(\boldsymbol{x}, t)$ and integrating it over a domain $\Omega$, where $\boldsymbol{v}$ satisfies the same boundary conditions as $\boldsymbol{u}$. The resulting scalar equation reads:

$$
\underbrace{\int_{\Omega} \frac{\partial \boldsymbol{u}}{\partial t} \cdot \boldsymbol{v} \mathrm{d} \Omega}_{\mathrm{I}}+\underbrace{\int_{\Omega} \nabla \cdot(\boldsymbol{c} \boldsymbol{u}) \cdot \boldsymbol{v} \mathrm{d} \Omega}_{\mathrm{II}}=-\underbrace{\int_{\Omega} \nabla p \cdot \boldsymbol{v} \mathrm{d} \Omega}_{\mathrm{III}}+\underbrace{v \int_{\Omega} \nabla^{2} \boldsymbol{u} \cdot \boldsymbol{v} \mathrm{d} \Omega}_{\mathrm{IV}} .
$$

Here $\boldsymbol{u}(\boldsymbol{x}, t)$ and $\boldsymbol{v}(\boldsymbol{x}, t)$ are elements of $L^{2}(\Omega)$, the space of square integrable functions on $\Omega$, with the following inner product:

$$
(\boldsymbol{u}, \boldsymbol{v}) \equiv \int_{\Omega} \boldsymbol{u} \cdot \boldsymbol{v} \mathrm{d} \Omega,
$$

implying the norm $\|\boldsymbol{u}\|^{2}=\int_{\Omega}|\boldsymbol{u}|^{2} \mathrm{~d} \Omega$. It should be stressed here that we have introduced an explicit distinction between the convecting quantity $\boldsymbol{c}$ and the convected quantity $\boldsymbol{u}$, see Eq. (8).

The separate terms in Eq. (8) will be rewritten with the divergence theorem to reveal a number of important properties. The second, convective, term is first written by employing the product rule as:

$$
\nabla \cdot(\boldsymbol{c u}) \cdot \boldsymbol{v}=(\boldsymbol{u} \cdot \boldsymbol{v}) \nabla \cdot \boldsymbol{c}+[(\boldsymbol{c} \cdot \nabla) \boldsymbol{u}] \cdot \boldsymbol{v} .
$$

Consequently we have

$$
[(\boldsymbol{c} \cdot \nabla) \boldsymbol{u}] \cdot \boldsymbol{v}=\nabla \cdot((\boldsymbol{u} \cdot \boldsymbol{v}) \boldsymbol{c})-(\boldsymbol{u} \cdot \boldsymbol{v}) \nabla \cdot \boldsymbol{c}-[(\boldsymbol{c} \cdot \nabla) \boldsymbol{v}] \cdot \boldsymbol{u} .
$$

When using $\nabla \cdot \boldsymbol{c}=0$, integrating over the domain, and applying integration by parts (divergence theorem) we obtain

$$
\text { II: } \int_{\Omega}[(\boldsymbol{c} \cdot \nabla) \boldsymbol{u}] \cdot \boldsymbol{v} \mathrm{d} \Omega=\int_{\partial \Omega}[(\boldsymbol{u} \cdot \boldsymbol{v}) \boldsymbol{c}] \cdot \boldsymbol{n} \mathrm{d} \Gamma-\int_{\Omega}[(\boldsymbol{c} \cdot \nabla) \boldsymbol{v}] \cdot \boldsymbol{u} \mathrm{d} \Omega .
$$

In case of periodic or no-penetration boundary conditions the boundary integral on the right-hand side vanishes, and the resulting equation can be written in terms of the inner product (9) as

$$
((\boldsymbol{c} \cdot \nabla) \boldsymbol{u}, \boldsymbol{v})=-(\boldsymbol{u},(\boldsymbol{c} \cdot \nabla) \boldsymbol{v}) .
$$

This relation expresses the skew-symmetry of the convective operator; it holds if $\nabla \cdot \boldsymbol{c}=0$ and boundary conditions are periodic or of no-penetration type. Denoting the convective operator by $(\boldsymbol{c} \cdot \nabla) \boldsymbol{u}=\boldsymbol{C}(\boldsymbol{c}) \boldsymbol{u}$ it can alternatively be written as

$$
\boldsymbol{C}(\boldsymbol{c})=-\boldsymbol{C}(\boldsymbol{c})^{*},
$$

where * denotes the Hermitian conjugate. Consequently, the convective term does not change the total energy of the flow:

$$
(\boldsymbol{C}(\boldsymbol{c}, \boldsymbol{u}), \boldsymbol{u})=0 .
$$

The third term, which expresses the work done by pressure forces, is written as

$$
\text { III: } \int_{\Omega} \nabla p \cdot \boldsymbol{v} \mathrm{d} \Omega=\int_{\partial \Omega} p \boldsymbol{v} \cdot \boldsymbol{n} \mathrm{d} \Gamma-\int_{\Omega} p \nabla \cdot \boldsymbol{v} \mathrm{d} \Omega .
$$

Again, in case of no penetration, periodic, or zero pressure boundary conditions this relation can be written as

$$
(\nabla p, \boldsymbol{v})=-(p, \nabla \cdot \boldsymbol{v})=0 .
$$

This equation expresses the compatibility relation between the divergence and gradient operator.

The last term, the viscous dissipation, is rewritten with $\nabla^{2} \boldsymbol{u} \cdot \boldsymbol{v}=\nabla \cdot\left(\boldsymbol{v} \cdot(\nabla \boldsymbol{u})^{T}\right)-\nabla \boldsymbol{v}: \nabla \boldsymbol{u},{ }^{1}$ leading to:

$$
\text { IV: } v \int_{\Omega} \nabla^{2} \boldsymbol{u} \cdot \boldsymbol{v} \mathrm{d} \Omega=v \int_{\partial \Omega} \boldsymbol{v} \cdot \frac{\partial \boldsymbol{u}}{\partial n} \mathrm{~d} \Gamma-v \int_{\Omega} \nabla \boldsymbol{v}: \nabla \boldsymbol{u} \mathrm{d} \Omega .
$$

\footnotetext{
1 The Frobenius product : is defined as $A: B \equiv \sum_{i} \sum_{j} A_{i j} B_{i j}=\operatorname{tr}\left(A^{T} B\right)$.
} 
Ignoring boundary terms and performing integration by parts once again one obtains the symmetry of the diffusive operator $\boldsymbol{D}(\boldsymbol{u})=\nabla^{2} \boldsymbol{u}:$

$$
\boldsymbol{D}=\boldsymbol{D}^{*} .
$$

To arrive at the equation for the evolution of the total kinetic energy we assume no-penetration or periodic boundary conditions and take $\boldsymbol{c}=\boldsymbol{u}, \boldsymbol{v}=\boldsymbol{u}$. The convective and pressure terms vanish, so the evolution equation for the kinetic energy $k=\frac{1}{2}\|\boldsymbol{u}\|^{2}$ becomes:

$$
\frac{\mathrm{d}}{\mathrm{d} t} \int_{\Omega} k \mathrm{~d} \Omega=v \int_{\partial \Omega} \boldsymbol{u} \cdot \frac{\partial \boldsymbol{u}}{\partial n} \mathrm{~d} \Gamma-v \int_{\Omega} \nabla \boldsymbol{u}: \nabla \boldsymbol{u} \mathrm{d} \Omega .
$$

In case of periodic boundary conditions the first term vanishes, so

$$
\frac{\mathrm{d} K}{\mathrm{~d} t}=-v(\nabla \boldsymbol{u}, \nabla \boldsymbol{u})
$$

where $K:=\int k \mathrm{~d} \Omega=\frac{1}{2}(\boldsymbol{u}, \boldsymbol{u})$ and the term on the right-hand side should be seen as a Frobenius product. Since $\nabla \boldsymbol{u}: \nabla \boldsymbol{u} \geqslant 0$, this equation shows that the total energy of the flow decreases due to viscous dissipation only; the convective and pressure terms do not contribute. If Eq. (21) is satisfied in a discrete sense, then the solution is bounded, independent of grid layout or time step.

\subsection{Pressure}

In the incompressible Navier-Stokes equations the pressure is a Lagrange multiplier which makes the velocity field divergence-free. There is no pressure evolution equation and as such it is not a conserved quantity like mass, momentum and energy. Furthermore, the original PDEs (1)-(2) do not contain boundary conditions for the pressure. However, an equation for the pressure with associated boundary conditions can be derived, and it is important to see if a discretization of the incompressible Navier-Stokes equations inherits the continuous properties of the pressure in a discrete sense. The pressure Poisson equation (PPE) is obtained by taking the time-derivative of the continuity equation and substituting the momentum equation [4]:

$$
\nabla^{2} p=\nabla \cdot \boldsymbol{N}(\boldsymbol{u})
$$

where $\boldsymbol{N}(\boldsymbol{u})=-(\boldsymbol{u} \cdot \nabla) \boldsymbol{u}+v \nabla^{2} \boldsymbol{u}$. On no-slip boundaries, where $\boldsymbol{u}=\boldsymbol{u}_{b}$, the boundary condition for the pressure is found by taking the normal component of the momentum equations at the boundary, i.e.

$$
\frac{\partial p}{\partial n}=\left(-\frac{\partial \boldsymbol{u}_{b}}{\partial t}+\boldsymbol{N}(\boldsymbol{u})\right) \cdot \boldsymbol{n} .
$$

\section{Conservation properties of the discrete equations}

In this section we will find discrete equivalents of the (continuous) equations of Section 2: conservation of mass (Section 3.2), conservation of momentum (Section 3.3), conservation of kinetic energy (Section 3.4) and the equation for the pressure (Section 3.7). The fourth order staggered grid discretization that will be used in these sections is first introduced in Section 3.1.

\subsection{Introduction}

We spatially discretize Eqs. (5)-(6) on a staggered Cartesian grid in two dimensions. A staggered grid yields a natural coupling between the divergence and gradient operator, which ensures that the pressure gradient does not contribute to the energy equation, as we will show later. The resulting semi-discrete system of equations reads:

$$
\begin{aligned}
& M u=0, \\
& \Omega \dot{u}+C(u) u=-G p+D u .
\end{aligned}
$$

We have switched from bold to normal font to distinguish between the continuous and discrete quantities. The unknowns $u$ and $p$ are point values of velocity and pressure in the center of the finite volumes (not volume averages), which depend on time only. Note that $u$ is used to denote both the vector with unknowns $u$ and $v$, and the $u$-component; the difference should be clear from the context. The diagonal matrix $\Omega=\operatorname{diag}\left(\Omega^{u}, \Omega^{v}\right)$ contains the finite volume sizes of the $u$ - and $v$-volumes (from the context confusion with $\Omega$ as arbitrary integration volume should be avoided). $M, C, G$ and $D$ represent the discrete divergence, convection, gradient and diffusion operators. To mimic the symmetry properties (14), (17), (19) in a discrete setting a discrete inner product has to be defined: 


$$
(u, v):=u^{T} \Omega v \text {. }
$$

This is a discrete analogue of Eq. (9).

Eqs. (24)-(25) are general expressions that are equally valid for the second order discretization of Harlow and Welch (HW) [6] and the fourth order discretization of Verstappen and Veldman (VV) [26]. The discretization of HW is constructed on a finite volume $\Omega_{1}=\Delta x \Delta y$, see Fig. 1(a) for the $u$-component. On non-uniform grids the velocity unknowns are still placed on the pressure volume boundaries. The boundaries of the velocity volumes are midway between two velocity unknowns, so that the velocity unknowns are not placed in the center of the velocity volumes. The discrete operators for the second order discretization are given by $M=M_{1}, \Omega=\Omega_{1}$, etc. The discretization of VV is constructed by combining the discretization on $\Omega_{1}$ with the discretization on a coarser finite volume $\Omega_{3}$. The discrete operators on $\Omega_{3}$ are indicated by $M_{3}, C_{3}$, etc. The coarse volumes are three times as large as the fine volumes (in each direction), see Fig. 1 (b). $\Omega_{1}$ and $\Omega_{3}$ are combined such that the leading order error term of the local truncation error cancels (Richardson extrapolation), resulting in a fourth order approximation. For example, the fourth order approximation to the $u$-component of the divergence equation reads

$$
\alpha_{1}\left(M_{1} u\right)_{i, j}-\left(M_{3} u\right)_{i, j}=\alpha_{1}\left(\bar{u}_{i, j}-\bar{u}_{i-1, j}\right)-\left(\overline{\bar{u}}_{i+1, j}-\overline{\bar{u}}_{i-2, j}\right) \text {, }
$$

where the face-integrated quantities $\bar{u}_{i, j}$ and $\overline{\bar{u}}_{i, j}$ are related to the point values $u_{i, j}$ via the midpoint rule:

$$
\begin{aligned}
& \bar{u}_{i, j}=\Delta y u_{i, j}, \\
& \overline{\bar{u}}_{i, j}=\alpha_{2} \Delta y u_{i, j} .
\end{aligned}
$$

In three dimensions an additional $\Delta z$ appears in these expressions. The bar $\overline{(.)}$ indicates integration in $x$ - or $y$-direction; this should be clear from the context. The coefficients $\alpha_{1}=3^{2+d}$ and $\alpha_{2}=3^{d-1}$, with $d$ the number of dimensions, are such that the leading order error term cancels. The effective finite volume size is given by $\Omega^{u}=\alpha_{1} \Omega_{1}^{u}-\Omega_{3}^{u}$; in case of a uniform grid, $\Omega_{3}^{u}=3^{d} \Omega_{1}^{u}$, so

$$
\Omega^{u}=3^{d}\left(3^{2}-1\right) \Omega_{1}^{u} .
$$

Upon division of the semi-discrete equations by this effective finite volume size one obtains the equations in 'finitedifference form'. For example, the divergence operator becomes

$$
\frac{1}{3^{d}\left(3^{2}-1\right) \Delta x \Delta y}\left(\alpha_{1} \Delta y\left(u_{i, j}-u_{i-1, j}\right)-\alpha_{2} \Delta y\left(u_{i+1, j}-u_{i-2, j}\right)\right)=\frac{9}{8} \frac{u_{i, j}-u_{i-1, j}}{\Delta x}-\frac{1}{8} \frac{u_{i+1, j}-u_{i-2, j}}{3 \Delta x} .
$$

The coefficients $\frac{9}{8}$ and $-\frac{1}{8}$ are the coefficients that appear in the finite difference method of Morinishi et al. [14] - the methods of Morinishi et al. and VV [26] are equivalent on uniform grids, and the analysis presented in the next sections is therefore equally valid for the finite difference method. On a non-uniform mesh, Morinishi et al. use mesh-dependent interpolation and differentiation to get fourth order accuracy, but sacrifice energy conservation properties. Vasilyev [21] improves Morinishi's scheme by constructing the discretization in computational space, but does not distinguish the interpolation operators for the convecting and convected quantities. Such a distinction is made by Ham et al. [5] (second order) and VV (fourth order), which leads to fully conservative discretization methods.

To make this paper self-contained we have detailed the spatial discretization at interior points in Appendix A. In the next sections we will focus on the spatial discretization near boundaries and investigate whether they satisfy discrete equivalents of the continuous identities expressing conservation of mass, momentum and energy.

\subsection{Mass}

We will investigate if the second and fourth order discretizations satisfy a discrete equivalent of

$$
\int_{\Omega} \nabla \cdot \boldsymbol{u} \mathrm{d} \Omega=\int_{\partial \Omega} \boldsymbol{u} \cdot \boldsymbol{n} \mathrm{d} \Gamma=0
$$

\subsubsection{Second order}

It suffices to focus on the fluxes of the $u$-component (in $x$-direction). In matrix notation the discrete divergence operator, Eq. (A.1), reads

$$
M_{1}^{u} \bar{u}=\left[\begin{array}{ccccc}
-1 & 1 & & \\
& -1 & 1 & \\
& & \ddots & \ddots &
\end{array}\right]\left[\begin{array}{c}
\bar{u}_{b, j} \\
\bar{u}_{1, j} \\
\bar{u}_{2, j} \\
\vdots
\end{array}\right]
$$




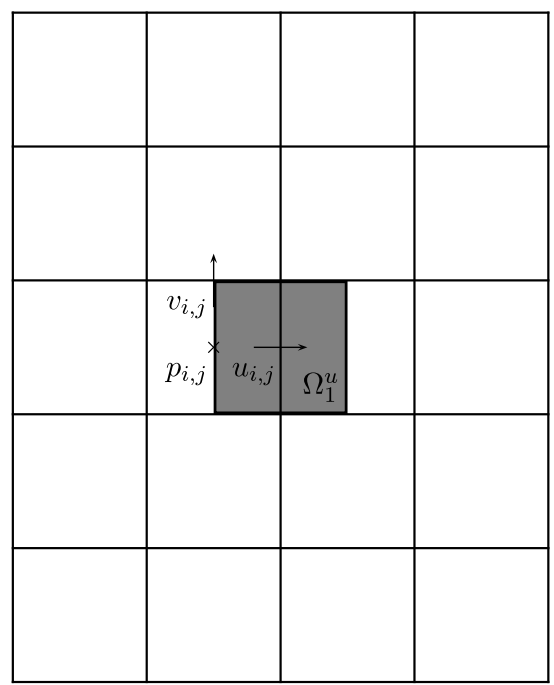

(a) fine

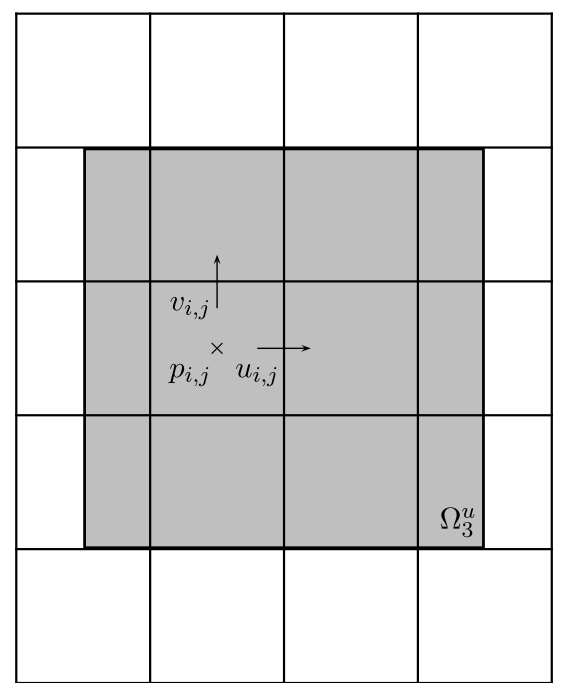

(b) coarse

Fig. 1. Fine and coarse finite volumes centered around $u_{i, j}$.

see Fig. 2(b). Discrete global mass conservation is found by summing the contribution of all finite volumes in $x$-direction (taking the column sum of (33)):

$$
1^{T} M_{1}^{u} \bar{u}=-\bar{u}_{b, j}+\text { (right boundary contribution). }
$$

Here $1^{T}$ denotes a row vector with entries one. Eq. (34) is the discrete equivalent of (32). Since we have a finite volume formulation, it is preferable to use the exact integral $\bar{u}_{b, j}$ in the discretization of the continuity equation, so that no discretization error is introduced in this equation. However, this integral value might not always be available (e.g. experimental data). When the midpoint rule (28) is employed for $\bar{u}_{b, j}$, and $u_{b, j}$ is not constant along a boundary, an $\mathcal{O}\left(h^{2}\right)$ error is introduced in this equation, and it does not evaluate to zero (like (32)). Fortunately, flow problems with an inflow boundary (non-zero $u_{b, j}$ ) necessarily include an outflow boundary, where the velocity adapts itself such that (34) evaluates exactly to 0 .

\subsubsection{Fourth order}

The divergence operator for the fourth order method is given by Eq. (A.9). To investigate the boundary contribution we evaluate (A.9) for $i=1$, i.e. the finite volume around $p_{1, j}$. Fig. 2(b) shows that the coarse volume extends over the physical boundary. We can focus on $M_{3}$. The choice for $\overline{\bar{u}}_{-1, j}$ should be such that the resulting divergence operator satisfies a summation-by-parts property. By taking the second order extrapolation

$$
\overline{\bar{u}}_{-1, j}=2 \overline{\bar{u}}_{b, j}-\overline{\bar{u}}_{1, j},
$$

the resulting divergence operator reads

$$
M_{3}^{u} \overline{\bar{u}}=\left[\begin{array}{cccccc}
-2 & 1 & 1 & & \\
-1 & & & 1 & \\
& -1 & & & 1 \\
& \ddots & \ddots & \ddots & \ddots &
\end{array}\right]\left[\begin{array}{c}
\overline{\bar{u}}_{b, j} \\
\overline{\bar{u}}_{1, j} \\
\overline{\bar{u}}_{2, j} \\
\overline{\bar{u}}_{3, j} \\
\vdots
\end{array}\right],
$$

and the discrete equivalent of (32) is

$$
1^{T} M_{3}^{u} \overline{\bar{u}}=-3 \overline{\bar{u}}_{b, j}+\text { (right boundary contribution). }
$$

After employing (29), a factor 9 results, which is due to coarse grid volumes being $9 \times$ larger than the fine grid volumes (in two dimensions). In other words: each fine grid volume is covered by 9 different coarse volumes. We note that with the boundary condition for the divergence operator suggested in VV [26],

$$
\overline{\bar{u}}_{-1, j}=\overline{\bar{u}}_{1, j}
$$

the summation-by-parts property (37) is not satisfied. 


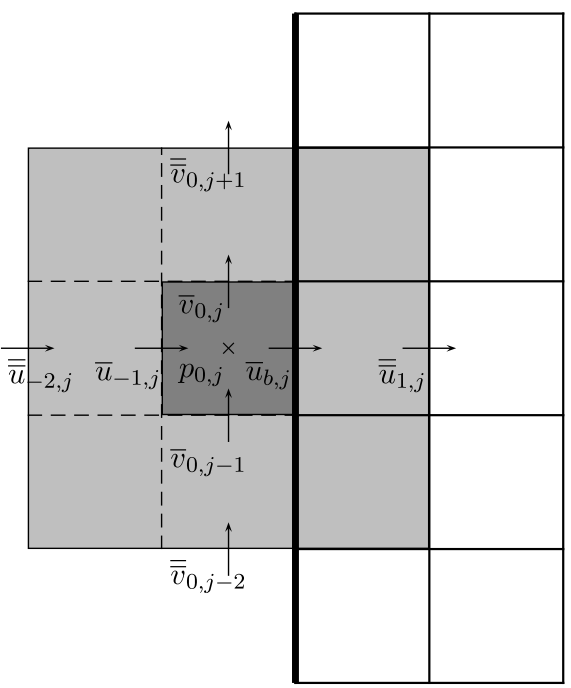

(a) $i=0$

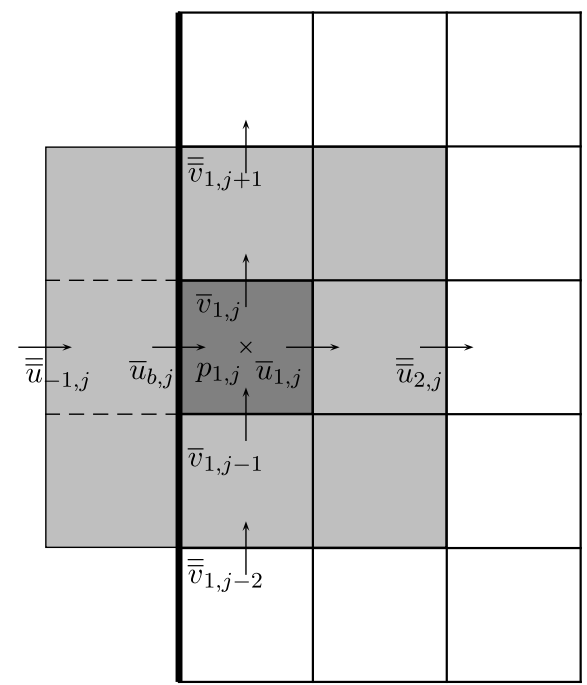

(b) $i=1$

Fig. 2. Boundary conditions divergence operator.

In Section 3.4.2 the divergence of a coarse volume centered around the ghost point $p_{0, j}$ will be needed in the discretization of the convective terms (see Fig. 2(a)):

$$
(M u)_{0, j}=\alpha_{1}\left(\bar{u}_{b, j}+\bar{v}_{0, j}-\bar{u}_{-1, j}-\bar{v}_{0, j-1}\right)-\left(\overline{\bar{u}}_{1, j}+\overline{\bar{v}}_{0, j+1}-\overline{\bar{u}}_{-2, j}-\overline{\bar{v}}_{0, j-2}\right) .
$$

This divergence is not enforced to be zero by the divergence operator. However, by properly choosing the ghost values it can be expressed in terms of $(M u)_{1, j}$, the divergence of the volume centered around $p_{1, j}$. We take, like (35) (see also [14]),

$$
\begin{aligned}
& \overline{\bar{u}}_{-2, j}=2 \overline{\bar{u}}_{b, j}-\overline{\bar{u}}_{2, j}, \\
& \bar{u}_{-1, j}=2 \bar{u}_{b, j}-\bar{u}_{1, j} .
\end{aligned}
$$

The linear extrapolation given by (41) leads to $\left(\frac{\partial u}{\partial x}\right)_{-1 / 2, j}=\left(\frac{\partial u}{\partial x}\right)_{1 / 2, j}$. Consequently, for mass conservation one requires $\left(\frac{\partial v}{\partial y}\right)_{-1 / 2, j}=\left(\frac{\partial v}{\partial y}\right)_{1 / 2, j}$, which is accomplished by choosing Neumann-type conditions for $v$ :

$$
\begin{aligned}
& \overline{\bar{v}}_{0, j}=\overline{\bar{v}}_{1, j}, \\
& \bar{v}_{0, j}=\bar{v}_{1, j} .
\end{aligned}
$$

With these conditions Eq. (39) can be written as

$$
\begin{aligned}
(M u)_{0, j} & =\alpha_{1}\left(\bar{u}_{b, j}+\bar{v}_{0, j}-\bar{u}_{-1, j}-\bar{v}_{0, j-1}\right)-\left(\overline{\bar{u}}_{1, j}+\overline{\bar{v}}_{0, j+1}-\overline{\bar{u}}_{-2, j}-\overline{\bar{v}}_{0, j-2}\right) \\
& =\alpha_{1}\left(\bar{u}_{1, j}+\bar{v}_{1, j}-\bar{u}_{b, j}-\bar{v}_{1, j-1}\right)-\left(\overline{\bar{u}}_{2, j}+\overline{\bar{v}}_{1, j+1}+\overline{\bar{u}}_{1, j}-\overline{\bar{v}}_{1, j-2}-2 \overline{\bar{u}}_{b, j}\right)=(M u)_{1, j}=0 .
\end{aligned}
$$

Once the boundary conditions for the divergence operator are chosen, the gradient operator for the pressure is fixed by requiring a discrete equivalent of Eq. (16), as will be discussed in Section 3.4. The boundary conditions for the pressure should therefore not be set separately from those for the velocity (in contrast to what is done in [14]). The boundary conditions for the divergence operator also influence the boundary treatment of the convective operator. This will be further detailed in Section 3.4.

\subsection{Momentum}

In this section we investigate the discrete equivalents of the following identities:

$$
\begin{aligned}
& \int_{\Omega}(\boldsymbol{u} \cdot \nabla) \boldsymbol{u} \mathrm{d} \Omega=\int_{\partial \Omega} \boldsymbol{u u} \cdot \boldsymbol{n} \mathrm{d} \Gamma, \\
& \int_{\Omega} \nabla p \mathrm{~d} \Omega=\int_{\partial \Omega} p \boldsymbol{n} \mathrm{d} \Gamma,
\end{aligned}
$$




$$
\int_{\Omega} \nabla^{2} \boldsymbol{u} \mathrm{d} \Omega=\int_{\partial \Omega} \frac{\partial \boldsymbol{u}}{\partial n} \mathrm{~d} \Gamma
$$

In contrast to the pressure finite volumes, the finite volumes associated with the $u$-and $v$-velocity components do not cover the entire computational domain (see for example Fig. 3(a)). In order to mimic (45) and (46) discretely, we propose a new approach that includes a discretization of the boundary volumes. This allows us to perform a discrete summation over the entire domain. Even though this discretization is not needed in practical computations, it is necessary to study the boundary contributions to the energy equation (Section 3.4). An alternative approach, which does not require the discretization of the boundary volumes, follows by writing (45) and (46) for the domain covered by the interior finite volumes (instead of the complete computational domain), but this approach is less suitable for the fourth order discretization.

\subsubsection{Second order}

We start again with the second order discretization to provide insight into the derivation of the fourth order method. In the interior the discretization conserves momentum since the fluxes of neighboring finite volumes cancel (the telescoping property of the finite volume method); see Appendix A.1. The conserved quantities are

$$
1^{T} \Omega^{u} u, 1^{T} \Omega^{v} v
$$

Near a vertical boundary we introduce the discretization of a "half boundary volume (the hatched area shown in Fig. 3(a)), so that the entire computational domain is covered. This discretization is taken such that global momentum is conserved:

$$
\left(\Omega_{1}^{u}\right)_{b, j} \frac{\mathrm{d} u_{b, j}}{\mathrm{~d} t}+\bar{u}_{1 / 2, j} u_{1 / 2, j}-\bar{u}_{b, j} u_{b, j}=-\left(\bar{p}_{1 j}-\bar{p}_{b, j}\right)+v\left(\left(\frac{\overline{\partial u}}{\partial x}\right)_{1 / 2, j}-\left(\frac{\overline{\partial u}}{\partial x}\right)_{b, j}\right) .
$$

We have left out the vertical fluxes, because they cancel upon summation in $y$-direction. After summation in $x$-direction we obtain:

$$
\begin{aligned}
1^{T} C_{1}^{u}(\bar{u}, \bar{v}) u & =-\bar{u}_{b, j} u_{b, j}+(\text { right boundary contribution }), \\
1^{T} G_{1}^{u} \bar{p} & =-\bar{p}_{b, j}+(\text { right boundary contribution }), \\
1^{T} \Delta_{1}^{u}\left(\frac{\overline{\partial u}}{\partial x}\right) & =-\left(\frac{\overline{\partial u}}{\partial x}\right)_{b, j}+\text { (right boundary contribution) } .
\end{aligned}
$$

These are the discrete counterparts of the $u$-component of equations (45)-(47). Face-integrated quantities such as $\bar{u}$ and $\bar{p}$ are approximated by the midpoint method, which is an $\mathcal{O}\left(h^{2}\right)$ approximation to the exact integral value. The discrete summation-by-parts expressions (50) and (51) do therefore not mimic the continuous relations exactly, except if the boundary values are constant or varying linearly along the boundary. One particular and important example is $u_{b, j}=0$, when the discrete convective terms do not contribute to the global momentum balance, and (50) mimics (45) exactly in a discrete sense.

The finite volumes of the $v$-component are aligned with a vertical boundary, so no boundary volume is necessary. The discretization of the first volume reads (leaving out vertical fluxes):

$$
\left(\Omega_{1}^{v}\right)_{1, j} \frac{\mathrm{d} v_{1, j}}{\mathrm{~d} t}+\bar{u}_{1, j+1 / 2} v_{3 / 2, j}-\bar{u}_{b, j+1 / 2} v_{b, j}=v\left(\left(\frac{\overline{\partial v}}{\partial x}\right)_{3 / 2, j}-\left(\frac{\overline{\partial v}}{\partial x}\right)_{b, j}\right) .
$$

The discrete equivalent of the $v$-component of (45) is

$$
1^{T} C_{1}^{v}(\bar{u}, \bar{v}) v=-\bar{u}_{b, j+1 / 2} v_{b, j}+\text { (right boundary contribution), }
$$

and similarly (47) becomes

$$
\left.1^{T} \Delta_{1}^{v}\left(\frac{\overline{\partial v}}{\partial x}\right)=-\left(\frac{\overline{\partial v}}{\partial x}\right)_{b, j}+\text { (right boundary contribution }\right) .
$$

The second order discretization of the convective terms does not require the imposition of ghost values. The diffusive gradients $\frac{\partial u}{\partial x}$ and $\frac{\partial v}{\partial x}$ are still to be expressed in terms of $u$ and $v$. This will be done in section 3.4, in such a way that the diffusive terms are dissipative.

The analysis near a horizontal boundary can be performed in a similar fashion. 


\subsubsection{Fourth order}

In the interior, conservation of momentum is obtained as for the second order case, although the cancellation of interior fluxes for the coarse volumes takes place over a distance of $3 \Delta x$ : the flux through the right face of a coarse volume around $u_{i, j}$ cancels with the flux through the left face of a coarse volume around $u_{i+3, j}$. The conserved quantity is $1^{T}\left(\alpha_{1} \Omega_{1}^{u}-\Omega_{3}^{u}\right) u$. Note that $1^{T} \Omega_{1}^{u} 1=\int \mathrm{d} \Omega=|\Omega|$, and that $1^{T} \Omega_{3}^{u} 1=9|\Omega|$ : every small volume is covered by 9 different coarse volumes. The total momentum conserved by the discretization is therefore not the discrete analogue of the single volume integral $\int_{\Omega} u \mathrm{~d} \Omega$, but the analogue of a combination of two instead.

The implied discretization at the boundary is more involved than in the second order case. It is necessary to introduce boundary volumes at $i=1, i=2$ and $i=3$, see Figs. 3(b)-3(d), which are taken such that the entire domain is covered completely by finite volumes. The boundary volumes are such that on each coarse grid discretization (I, II and III) a summation-by-parts identity is satisfied:

$$
\begin{aligned}
& \text { I: } \quad-\left(\Omega_{3}^{u}\right)_{\mathrm{I}} \frac{\mathrm{d} u_{b, j}}{\mathrm{~d} t}-\left(\overline{\bar{u}}_{b, j} u_{b, j}-\overline{\bar{u}}_{-1 / 2, j} u_{-1 / 2, j}\right)=-\left(\overline{\bar{p}}_{0, j}-\overline{\bar{p}}_{b, j}\right)-v\left(\left(\frac{\overline{\overline{\partial u}}}{\partial x}\right)_{b, j}-\left(\frac{\overline{\overline{\partial u}}}{\partial x}\right)_{-1 / 2, j}\right), \\
& \text { II: } \quad\left(\Omega_{3}^{u}\right)_{\mathrm{II}} \frac{\mathrm{d} u_{b, j}}{\mathrm{~d} t}+\overline{\bar{u}}_{1 / 2, j} u_{1 / 2, j}-\overline{\bar{u}}_{b, j} u_{b, j}=-\left(\overline{\bar{p}}_{1, j}-\overline{\bar{p}}_{b, j}\right)+v\left(\left(\frac{\overline{\overline{\partial u}}}{\partial x}\right)_{1 / 2, j}-\left(\frac{\overline{\overline{\partial u}}}{\partial x}\right)_{b, j}\right), \\
& \text { III: } \quad\left(\Omega_{3}^{u}\right)_{\mathrm{III}} \frac{\mathrm{d} u_{b, j}}{\mathrm{~d} t}+\overline{\bar{u}}_{3 / 2, j} u_{3 / 2, j}-\overline{\bar{u}}_{b, j} u_{b, j}=-\left(\overline{\bar{p}}_{2, j}-\overline{\bar{p}}_{b, j}\right)+v\left(\left(\frac{\overline{\overline{\partial u}}}{\partial x}\right)_{3 / 2, j}-\left(\frac{\overline{\overline{\partial u}}}{\partial x}\right)_{b, j}\right) .
\end{aligned}
$$

Here we have focused again on the horizontal fluxes. The boundary volumes $\left(\Omega_{3}^{u}\right)_{\mathrm{I}},\left(\Omega_{3}^{u}\right)_{\text {II }}$ and $\left(\Omega_{3}^{u}\right)_{\mathrm{III}}$ are illustrated by the hatched areas. The implied discretization of the boundary volume is given by the sum of the three expressions above:

$$
\begin{aligned}
\left(\Omega_{3}^{u}\right)_{b, j} \frac{\mathrm{d} u_{b, j}}{\mathrm{~d} t} & +\overline{\bar{u}}_{-1 / 2, j} u_{-1 / 2, j}+\overline{\bar{u}}_{1 / 2, j} u_{1 / 2, j}+\overline{\bar{u}}_{3 / 2, j} u_{3 / 2, j}-3 \overline{\bar{u}}_{b, j} u_{b, j} \\
= & -\left(\overline{\bar{p}}_{0, j}+\overline{\bar{p}}_{1, j}+\overline{\bar{p}}_{2, j}-3 \overline{\bar{p}}_{b, j}\right)+v\left(\left(\frac{\overline{\overline{\partial u}}}{\partial x}\right)_{-1 / 2, j}+\left(\frac{\overline{\overline{\partial u}}}{\partial x}\right)_{1 / 2, j}+\left(\frac{\overline{\overline{\partial u}}}{\partial x}\right)_{3 / 2, j}-3\left(\frac{\overline{\overline{\partial u}}}{\partial x}\right)_{b, j}\right),
\end{aligned}
$$

where

$$
\left(\Omega_{3}^{u}\right)_{b, j}=-\left(\Omega_{3}^{u}\right)_{\mathrm{I}}+\left(\Omega_{3}^{u}\right)_{\mathrm{II}}+\left(\Omega_{3}^{u}\right)_{\mathrm{III}}=\left(\Omega_{3}^{u}\right)_{\mathrm{III}} .
$$

Summing the contribution of all (interior and boundary) fluxes gives

$$
\begin{aligned}
1^{T} C_{3}^{u}(\overline{\bar{u}}, \overline{\bar{v}}) u & =-3 \overline{\bar{u}}_{b, j} u_{b, j}+(\text { right boundary contribution }), \\
1^{T} G_{3}^{u} p & =-3 \overline{\bar{p}}_{b, j}+(\text { right boundary contribution }),
\end{aligned}
$$

and

$$
1^{T} \Delta_{3}^{u}\left(\frac{\overline{\overline{\partial u}}}{\partial x}\right)=-3\left(\frac{\overline{\overline{\partial u}}}{\partial x}\right)_{b, j}+(\text { right boundary contribution })
$$

the discrete counterparts of Eqs. (45)-(47).

For the $v$-component near a vertical boundary a different technique has to be applied. When introducing, like for the $u$-component, a ('negative') boundary volume for $i=1$ and a ('positive') boundary volume for $i=3$, such that on each coarse grid momentum is conserved, one would obtain a discretization for a boundary volume with zero size, which is not appropriate when studying the energy equation. Instead, we construct the discretization for $v_{1, j}$ as the sum of the boundary volumes of coarse grid II and III, see Fig. 4:

$$
\left(\Omega_{3}^{v}\right)_{1, j} \frac{\mathrm{d} v_{1, j}}{\mathrm{~d} t}+\overline{\bar{u}}_{1, j+1 / 2} v_{3 / 2, j}+\overline{\bar{u}}_{2, j+1 / 2} v_{5 / 2, j}-2 \overline{\bar{u}}_{b, j+1 / 2} v_{b, j}=v\left(\left(\frac{\overline{\overline{\partial v}}}{\partial x}\right)_{5 / 2, j}+\left(\frac{\overline{\overline{\partial v}}}{\partial x}\right)_{3 / 2, j}-2\left(\frac{\overline{\overline{\partial v}}}{\partial x}\right)_{b, j}\right),
$$

where $\left(\Omega_{3}^{v}\right)_{1, j}=\Omega_{\mathrm{II}}+\Omega_{\mathrm{III}}$. In contrast to Eq. (59), $v_{1, j}$ in Eq. (64) is an unknown in the system of equations and is solved for. Summation in $x$-direction gives

$$
1^{T} C_{3}^{v}(\overline{\bar{u}}, \overline{\bar{v}}) v=-3 \overline{\bar{u}}_{b, j+1 / 2} v_{b, j}+\text { (right boundary contribution), }
$$




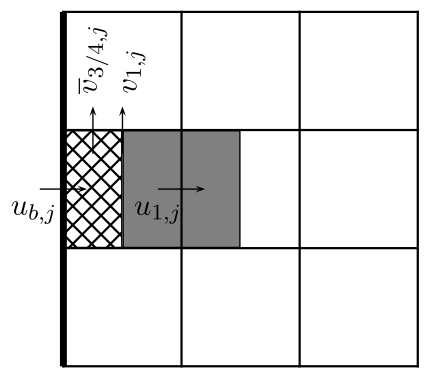

(a) fine grid

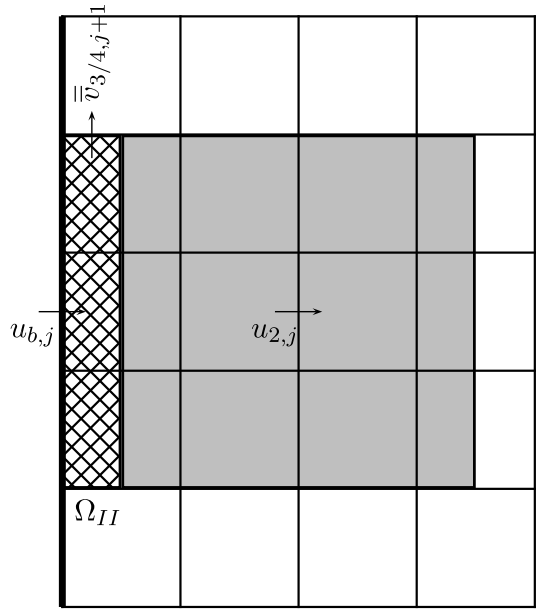

(c) coarse grid II, $i=2$

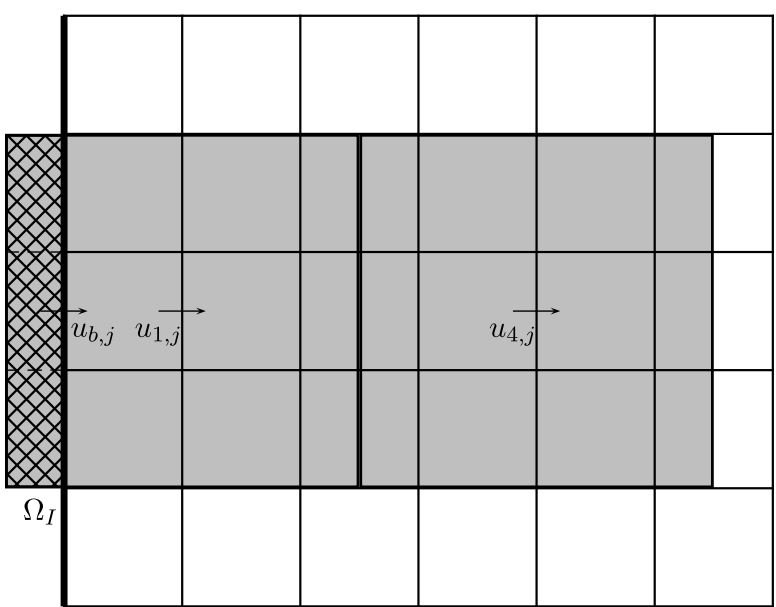

(b) coarse grid I: $i=1, i=4$

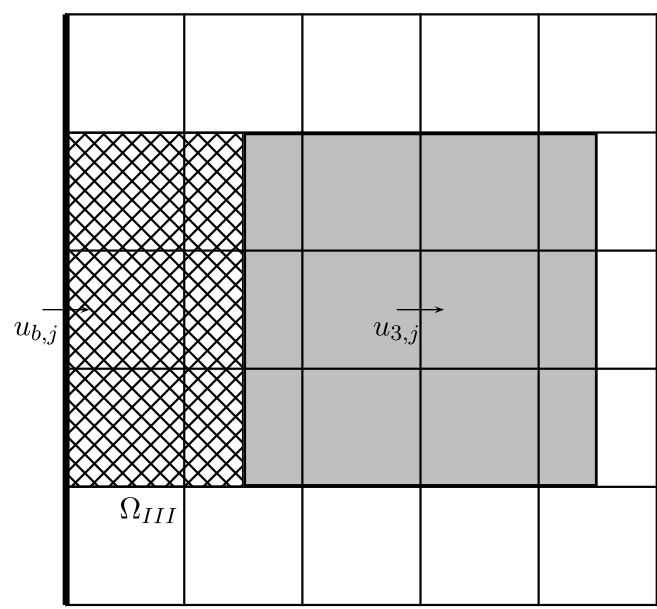

(d) coarse grid III, $i=3$

Fig. 3. Momentum conservation near a boundary; $u$-momentum.

$$
1^{T} \Delta_{3}^{v}\left(\frac{\overline{\overline{\partial v}}}{\partial x}\right)=-3\left(\frac{\overline{\overline{\partial v}}}{\partial x}\right)_{b, j}+(\text { right boundary contribution }),
$$

which are the discrete equivalents of the $v$-component of (45) and (47).

Finally it may be noted that momentum is conserved independently of the treatment of the ghost values (such as $\overline{\bar{p}}_{0, j}$, $u_{-1 / 2, j}$, etc.).

\subsection{Kinetic energy}

In this section we investigate if Eqs. (12), (16) and (18), with $\boldsymbol{v}$ replaced by $\boldsymbol{u}$, i.e.

$$
\begin{aligned}
\int_{\Omega}[(\boldsymbol{u} \cdot \nabla) \boldsymbol{u}] \cdot \boldsymbol{u} \mathrm{d} \Omega & =\frac{1}{2} \int_{\partial \Omega}[(\boldsymbol{u} \cdot \boldsymbol{u}) \boldsymbol{u}] \cdot \boldsymbol{n} \mathrm{d} \Gamma, \\
\int_{\Omega} \nabla p \cdot \boldsymbol{u} \mathrm{d} \Omega & =\int_{\partial \Omega} p \boldsymbol{u} \cdot \boldsymbol{n} \mathrm{d} \Gamma-\int_{\Omega} p \nabla \cdot \boldsymbol{u} \mathrm{d} \Omega, \\
\int_{\Omega} \nabla^{2} \boldsymbol{u} \cdot \boldsymbol{u} \mathrm{d} \Omega & =\int_{\partial \Omega} \boldsymbol{u} \cdot \frac{\partial \boldsymbol{u}}{\partial n} \mathrm{~d} \Gamma-\int_{\Omega} \nabla \boldsymbol{u}: \nabla \boldsymbol{u} \mathrm{d} \Omega,
\end{aligned}
$$

can be satisfied in a discrete sense. The total kinetic energy that should be conserved by the discretization in case of inviscid flow (according to Eq. (21)) is obtained by multiplying the $u$-momentum equation with $u$, the $v$-momentum equation with $v$, like in the continuous case: 


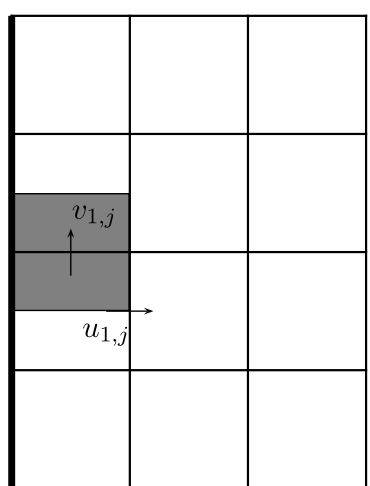

(a) fine grid

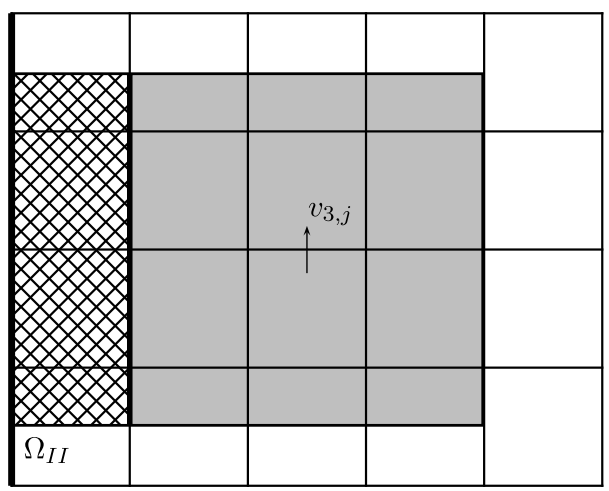

(c) coarse grid II, $i=3$

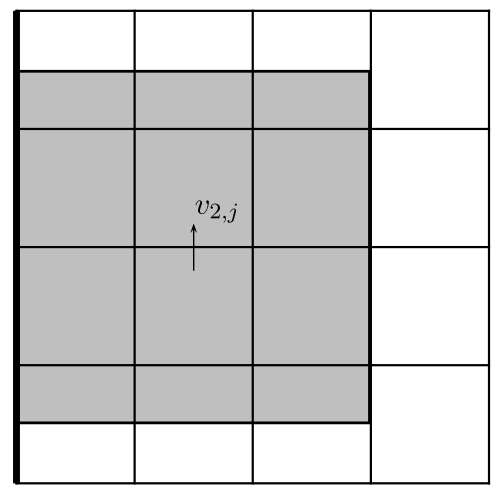

(b) coarse grid I: $i=2$

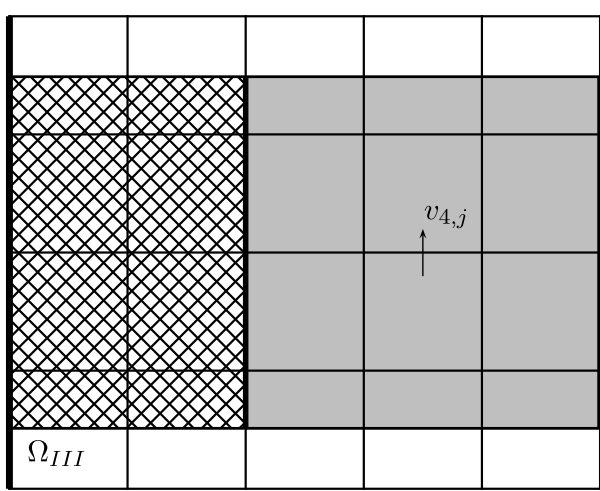

(d) coarse grid III, $i=4$

Fig. 4. Momentum conservation near a boundary; v-momentum.

$$
K:=\frac{1}{2}\left(u, \Omega^{u} u\right)+\frac{1}{2}\left(v, \Omega^{v} v\right) .
$$

\subsubsection{Second order}

Eq. (67) indicates that in the interior the convective terms do not contribute to the kinetic energy. This can be proven in a discrete sense by showing that the divergence form (A.3) can be written in skew-symmetric form. Indeed, if $C$ is skew-symmetric, then $u^{T} C(c) u=0$ for any $u$. When constant (grid-independent) weights are used to interpolate $\bar{u}$ and $\bar{v}$, Eq. (A.3) can be written in the skew-symmetric form

$$
\begin{aligned}
\left(C_{1}^{u}(\bar{u}, \bar{v}) u\right)_{i, j}= & \bar{u}_{i+1 / 2, j} \frac{1}{2} u_{i+1, j}-\bar{u}_{i-1 / 2, j} \frac{1}{2} u_{i-1, j}+\bar{v}_{i+1 / 2, j} \frac{1}{2} u_{i, j+1}-\bar{v}_{i+1 / 2, j-1} \frac{1}{2} u_{i, j-1} \\
& +\frac{1}{2} u_{i, j}\left[\frac{1}{2}\left(\bar{u}_{i+1, j}-\bar{u}_{i, j}+\bar{v}_{i+1, j}-\bar{v}_{i+1, j-1}\right)+\frac{1}{2}\left(\bar{u}_{i, j}-\bar{u}_{i-1, j}+\bar{v}_{i, j}-\bar{v}_{i, j-1}\right)\right]
\end{aligned}
$$

The term in square brackets features the sum of the divergence of the pressure volumes to the left and right of $u_{i, j}$ : the diagonal coefficient of the convective operator is therefore zero, like in the continuous case (Eq. (11)).

Near boundaries the convective discretization should not be skew-symmetric in case energy flows in or out of the domain, corresponding to the boundary integral in Eq. (12). We elaborate the discretization near a boundary, given by (49), where now the vertical fluxes are considered too:

$$
\left(C_{1}^{u}(\bar{u}, \bar{v}) u\right)_{b, j}=\bar{u}_{1 / 2, j} u_{1 / 2, j}-\bar{u}_{b, j} u_{b, j}+\bar{v}_{3 / 4, j} u_{b, j+1 / 2}-\bar{v}_{3 / 4, j-1} u_{b, j-1 / 2}
$$

These terms are used to rewrite the divergence form into skew-symmetric form. In matrix notation $C_{1}^{u}(\bar{u}, \bar{v})$ reads

$$
C_{1}^{u}(\bar{u}, \bar{v})=\left[\begin{array}{c|ccc}
-\bar{u}_{b, j}+\frac{1}{2} \bar{u}_{1 / 2, j}+\frac{1}{2}\left(\bar{v}_{3 / 4, j}-\bar{v}_{3 / 4, j-1}\right) & \frac{1}{2} \bar{u}_{1 / 2, j} & & \\
-\frac{1}{2} \bar{u}_{1 / 2, j} & 0 & \frac{1}{2} \bar{u}_{3 / 2, j} & \\
& -\frac{1}{2} \bar{u}_{3 / 2, j} & 0 & \ddots \\
& & \ddots
\end{array}\right] .
$$


The first row represents the equation for $u_{b, j}$, the first column represents the contribution of $u_{b, j}$. The lower-right part of the matrix (which is indicated by the line) represents the internal discretization. The matrix $C_{1}^{u}(\bar{u}, \bar{v})$ is skew-symmetric except for its upper left entry, so the discrete version of (67) becomes

$$
u^{T} C_{1}^{u}(\bar{u}, \bar{v}) u=u_{b, j}^{2}\left(-\bar{u}_{b, j}+\frac{1}{2} \bar{u}_{1 / 2, j}+\frac{1}{2}\left(\bar{v}_{3 / 4, j}-\bar{v}_{3 / 4, j-1}\right)\right) .
$$

The vertical flux $\bar{v}_{3 / 4, j}$ should be computed as $\bar{v}_{3 / 4, j}=\frac{1}{2} \bar{v}_{1, j}$ since this leads to

$$
\begin{aligned}
u^{T} C_{1}^{u}(\bar{u}, \bar{v}) u & =-\frac{1}{2} u_{b, j}^{2} \bar{u}_{b, j}+\frac{1}{4} u_{b, j}^{2}\left(\bar{u}_{1, j}-\bar{u}_{b, j}+\bar{v}_{1, j}-\bar{v}_{1, j-1}\right) \\
& =-\frac{1}{2} u_{b, j}^{2} \bar{u}_{b, j}
\end{aligned}
$$

which is indeed the discrete equivalent of (67). Property (75) is only exact if $u_{b}$ is constant or varying linearly along a boundary; in all other cases it is an $\mathcal{O}\left(h^{2}\right)$ approximation to equation (67).

The convective matrix for the $v$-component reads

$$
C_{1}^{v}(\bar{u}, \bar{v}) v=\left[\begin{array}{ccc}
\frac{1}{2} \bar{u}_{1, j+1 / 2}+\frac{1}{2}\left(\bar{v}_{1, j+1 / 2}-\bar{v}_{1, j-1 / 2}\right) & \frac{1}{2} \bar{u}_{1, j+1 / 2} \\
-\frac{1}{2} \bar{u}_{1, j+1 / 2} & 0 & \frac{1}{2} \bar{u}_{2, j+1 / 2}
\end{array}\right]\left[\begin{array}{c}
v_{1} \\
v_{2} \\
\vdots
\end{array}\right]+\left[\begin{array}{c}
-\bar{u}_{b, j+1 / 2} v_{b, j} \\
0 \\
\vdots
\end{array}\right],
$$

and the contribution to the energy equation becomes

$$
v^{T} C_{1}^{v}(\bar{u}, \bar{v}) v=-\frac{1}{2} v_{1, j} \bar{u}_{b, j+1 / 2}\left(2 v_{b, j}-v_{1, j}\right)
$$

Surprisingly, this term does not evaluate to $-\frac{1}{2} v_{b, j}^{2} \bar{u}_{b, j+1 / 2}$, which one might have expected intuitively. However, upon Taylor expanding $v_{1, j}$ around $v_{b, j}$,

$$
v_{1, j}=v_{b, j}+\frac{1}{2} h\left(v_{x}\right)_{b, j}+\mathcal{O}\left(h^{2}\right)
$$

we see that

$$
\frac{1}{2} v_{1, j} \bar{u}_{b, j+1 / 2}\left(2 v_{b, j}-v_{1, j}\right)=\frac{1}{2} \bar{u}_{b, j+1 / 2} v_{b, j}^{2}+\mathcal{O}\left(h^{2}\right),
$$

which is, like (75), a second order approximation to the continuous boundary integral. However, in contrast to (75), this approximation is not exact for constant $u_{b}$ and $v_{b}$.

For the pressure gradient terms the discrete second order version of (68) reads (here we concentrate again on summing in $x$-direction):

$$
\begin{aligned}
u^{T} G_{1}^{u} \bar{p}+v^{T} G_{1}^{v} \bar{p}= & \bar{u}^{T} G_{1}^{u} p+\bar{v}^{T} G_{1}^{v} p \\
= & \bar{u}_{b, j}\left(p_{1, j}-p_{b, j}\right)+\bar{u}_{1, j}\left(p_{2, j}-p_{1, j}\right)+\cdots \\
& +\bar{v}_{1, j}\left(p_{1, j+1}-p_{1, j}\right)+\bar{v}_{1, j-1}\left(p_{1, j}-p_{1, j-1}\right)+\cdots \\
= & -\bar{u}_{b, j} p_{b, j}-p_{1, j} \underbrace{\left(\bar{u}_{1, j}-\bar{u}_{b, j}+\bar{v}_{1, j}-\bar{v}_{1, j-1}\right)}_{\left(M_{1} u\right)_{1, j}}-p_{2, j} \underbrace{(\ldots)}_{\left(M_{1} u\right)_{2, j}}+\cdots \\
= & -\bar{u}_{b, j} p_{b, j}+\text { (right boundary contribution). }
\end{aligned}
$$

In Eq. (81) we have switched from $\bar{p}$ to $\bar{u}$ : this is valid, even on non-uniform grids, since both face quantities are approximated using (28). In matrix notation the above result can be written as

$$
\left.u^{T} G_{1} \bar{p}=-\bar{u}_{b, j} p_{b, j}+\text { (right boundary contribution }\right)-p^{T} M_{1} \bar{u}
$$

In case of no-penetration or periodic boundary conditions one obtains the well-known symmetry relation between the divergence and the gradient operator:

$$
M_{1}=-G_{1}^{T}
$$


The contribution of the diffusive terms to the energy equation reads

$$
\begin{aligned}
u^{T} D_{1}^{u} u & =u_{b, j}\left(\left(\frac{\overline{\partial u}}{\partial x}\right)_{1 / 2, j}-\left(\frac{\overline{\partial u}}{\partial x}\right)_{b, j}\right)+u_{1, j}\left(\left(\frac{\overline{\partial u}}{\partial x}\right)_{3 / 2, j}-\left(\frac{\overline{\partial u}}{\partial x}\right)_{1 / 2, j}\right)+u_{2, j}(.)+\cdots \\
& =-u_{b, j}\left(\frac{\overline{\partial u}}{\partial x}\right)_{b, j}-\left(\frac{\overline{\partial u}}{\partial x}\right)_{1 / 2, j}\left(u_{1, j}-u_{b, j}\right)-\left(\frac{\overline{\partial u}}{\partial x}\right)_{3 / 2, j}\left(u_{2, j}-u_{1, j}\right)+\cdots
\end{aligned}
$$

With the following discretization of the first order derivatives,

$$
\left(\frac{\overline{\partial u}}{\partial x}\right)_{i+1 / 2, j}=\Delta y\left(\frac{u_{i+1, j}-u_{i, j}}{\Delta x}\right),
$$

Eq. (87) can be written as

$$
u^{T} D_{1}^{u} u=-u_{b, j}\left(\frac{\overline{\partial u}}{\partial x}\right)_{b, j}-\Delta x \Delta y\left(\left(\frac{u_{1, j}-u_{b, j}}{\Delta x}\right)^{2}+\left(\frac{u_{2, j}-u_{1, j}}{\Delta x}\right)^{2}+\cdots\right),
$$

which is the discrete equivalent of (69). The derivative at the boundary does not have to be specified since it is not needed in practical calculations.

The $v$-component can be treated in a similar fashion:

$$
\begin{aligned}
v^{T} D_{1}^{v} v & =v_{1, j}\left(\left(\frac{\overline{\partial v}}{\partial x}\right)_{3 / 2, j}-\left(\frac{\overline{\partial v}}{\partial x}\right)_{b, j}\right)+v_{2, j}\left(\left(\frac{\overline{\partial v}}{\partial x}\right)_{5 / 2, j}-\left(\frac{\overline{\partial v}}{\partial x}\right)_{3 / 2, j}\right)+v_{3, j}(.)+\cdots \\
& =-v_{1, j}\left(\frac{\overline{\partial v}}{\partial x}\right)_{b, j}-\Delta x \Delta y\left(\left(\frac{v_{2, j}-v_{1, j}}{\Delta x}\right)^{2}+\left(\frac{v_{3, j}-v_{2, j}}{\Delta x}\right)^{2}+\cdots\right) .
\end{aligned}
$$

By taking the approximation

$$
\left(\frac{\overline{\partial v}}{\partial x}\right)_{b, j}=\Delta y\left(\frac{v_{1, j}-v_{b, j}}{\frac{1}{2} \Delta x}\right)
$$

Eq. (90) can be written as

$$
v^{T} D_{1}^{v} v=-v_{b, j} \Delta y\left(\frac{v_{1, j}-v_{b, j}}{\frac{1}{2} \Delta x}\right)-\Delta x \Delta y\left(\frac{1}{2}\left(\frac{v_{1, j}-v_{b, j}}{\Delta x}\right)^{2}+\left(\frac{v_{2, j}-v_{1, j}}{\Delta x}\right)^{2}+\left(\frac{v_{3, j}-v_{2, j}}{\Delta x}\right)^{2}+\cdots\right),
$$

which is the discrete counterpart of Eq. (69). The approximation of the derivative at the boundary can be viewed as a centered approximation with ghost value $v_{0, j}=2 v_{b, j}-v_{1, j}$.

\subsubsection{Fourth order}

Like the second order scheme, the fourth order convective discretization (A.11) can be rewritten in skew-symmetric form. To that end, the diagonal coefficient is expressed in terms of the divergence of four surrounding finite volumes. For details we refer to [26].

Near boundaries this skew-symmetric form is used to derive the contribution to the energy equation. The analysis is detailed in Appendix B.1. An important point is the choice of the ghost values. Here the ghost values for the $u$-component at a vertical boundary are defined by a second-order extrapolation, for example $u_{-1, j}=2 u_{b, j}-u_{1, j}$. The ghost values for the $v$-component at a vertical boundary are given by Neumann type conditions, see (42), (43) and (B.15). This leads to

$$
\begin{aligned}
& u^{T} C_{3}^{u} u=-\frac{3}{2} \overline{\bar{u}}_{b, j} u_{b, j}^{2}+\mathcal{O}\left(h^{2}\right), \\
& v^{T} C_{3}^{v} v=-\frac{3}{2} \overline{\bar{u}}_{b, j+1 / 2} v_{b, j}^{2}+\mathcal{O}\left(h^{2}\right) .
\end{aligned}
$$

These equations reveal that the boundary integrals in (67) are not satisfied discretely (not even for constant boundary data) but contain an $\mathcal{O}\left(h^{2}\right)$ error. However, in the important case of $\overline{\bar{u}}_{b, j}=0$ the discretization mimics the continuous property (no energy contribution by the convective terms) exactly. At a horizontal boundary the same analysis applies.

The contribution of the pressure gradient to the energy equation for the fourth order method is given by

$$
\begin{aligned}
u^{T} G_{3}^{u} \overline{\bar{p}}+v^{T} G_{3}^{v} \overline{\bar{p}} & =\overline{\bar{u}}_{b, j}\left(p_{0, j}+p_{1, j}+p_{2, j}-3 p_{b, j}\right)+\overline{\bar{u}}_{1, j}\left(p_{3, j}-p_{0, j}\right)+\overline{\bar{u}}_{2, j}\left(p_{4, j}-p_{1, j}\right)+(v \text {-component }) \\
& =-3 \overline{\bar{u}}_{b, j} p_{b, j}+p_{0, j}\left(\overline{\bar{u}}_{1, j}-\overline{\bar{u}}_{b, j}\right)+p_{1, j}\left(\overline{\bar{u}}_{2, j}-\overline{\bar{u}}_{b, j}+\overline{\bar{v}}_{1, j+1}-\overline{\bar{v}}_{1, j-2}\right)+\cdots
\end{aligned}
$$




$$
\begin{aligned}
& =-3 \overline{\bar{u}}_{b, j} p_{b, j}+p_{1, j} \underbrace{\left(\overline{\bar{u}}_{2}+\overline{\bar{u}}_{1}-2 \overline{\bar{u}}_{b, j}+\overline{\bar{v}}_{1, j+1}-\overline{\bar{v}}_{1, j-2}\right)}_{\left(M_{3} u\right)_{1, j}}+\cdots \\
& =-3 \overline{\bar{u}}_{b, j} p_{b, j}+\text { (right boundary contribution). }
\end{aligned}
$$

The Neumann condition $p_{0, j}=p_{1, j}$ is necessary to write Eq. (96) as (97): the term in brackets in (97) then evaluates to 0 since it is the divergence of a coarse volume around $p_{1, j}$ (see Section 3.2.2). In short notation, the fourth order scheme satisfies

$$
\left.u^{T} G_{3} \overline{\bar{p}}=-3 \overline{\bar{u}}_{b, j} p_{b, j}+\text { (right boundary contribution }\right)-p^{T} M_{3} \overline{\bar{u}} .
$$

There are two important notes to be made regarding condition $p_{0, j}=p_{1, j}$. Firstly, this condition follows from the discretization of the divergence operator and by requiring (99). Consequently, for the discretization of the interior points, no explicit pressure boundary conditions need to be specified separately from those for the velocity, as was the case for the second order discretization. Notice that pressure boundary conditions were not specified for the continuous problem either. Secondly, the 'implied' discrete Neumann condition is not homogeneous, in contrast to what $p_{0, j}=p_{1, j}$ suggests. This will be detailed in Section 3.7. We note that boundary condition (38), as suggested in [26], effectively leads to a Dirichlet condition for the pressure. Near solid walls this results in an artificial boundary layer in the pressure.

Lastly, we discuss the contribution of the diffusive terms to the energy equation. In Appendix B.2 we show that for the fourth order scheme:

$$
\begin{aligned}
u^{T} D^{u} u= & -\left(\frac{\overline{\partial u}}{\partial x}\right)_{b, j}\left(\alpha_{1}-9\right) u_{b, j}+\cdots-72 \Delta x \Delta y\left[\left(\frac{9}{8} \frac{u_{1, j}-u_{b, j}}{\Delta x}-\frac{1}{8} \frac{u_{2, j}+u_{1, j}-2 u_{b, j}}{3 \Delta x}\right)^{2}\right. \\
& \left.+\left(\frac{9}{8} \frac{u_{2, j}-u_{1, j}}{\Delta x}-\frac{1}{8} \frac{u_{3, j}-u_{b, j}}{3 \Delta x}\right)^{2}+\cdots\right] .
\end{aligned}
$$

This is the discrete equivalent of Eq. (69), with the first term representing the boundary integral, and the second term the volume integral.

\subsection{Extension to higher order}

The boundary conditions that are derived in Sections 3.2-3.4 are all based on first or second order extrapolations. This limits the local truncation error of the fourth order scheme, as will be shown in Section 4. In this section we will investigate if the ghost values can be obtained by higher order extrapolations while still satisfying discrete equivalents of the continuous properties derived in Section 2. We start with the divergence operator. Eq. (35) can be generalized to

$$
\overline{\bar{u}}_{-1, j}=\delta_{0} \overline{\bar{u}}_{b, j}+\delta_{1} \overline{\bar{u}}_{1, j}+\delta_{2} \overline{\bar{u}}_{2, j}+\delta_{3} \overline{\bar{u}}_{3, j}+\cdots,
$$

hence the divergence operator becomes

$$
M_{3}^{u} \overline{\bar{u}}=\left[\begin{array}{ccccc}
-\delta_{0} & -\delta_{1} & 1-\delta_{2} & -\delta_{3} & \\
-1 & & & 1 & \\
& -1 & & & 1 \\
& & \ddots & \ddots & \ddots
\end{array}\right]\left[\begin{array}{c}
\overline{\bar{u}}_{b, j} \\
\overline{\bar{u}}_{1, j} \\
\overline{\bar{u}}_{2, j} \\
\overline{\bar{u}}_{3, j} \\
\vdots
\end{array}\right] .
$$

The only solution that satisfies the summation-by-parts identity (37) is $\delta_{0}=2, \delta_{1}=-1, \delta_{2}=\delta_{3}=\cdots=0$, which equals boundary condition (35). The other boundary conditions derived in Section 3.2, Eqs. (40)-(43), cannot be changed following similar reasoning.

We can also try more general formulations for $u_{-1 / 2, j}$ and $u_{1 / 2, j}$ (see Eqs. (B.1)-(B.2)):

$$
\begin{aligned}
u_{-1 / 2, j} & =\beta_{0} u_{b, j}+\beta_{1} u_{1, j}+\beta_{2} u_{2, j}+\beta_{3} u_{3, j}+\cdots, \\
u_{1 / 2, j} & =\gamma_{0} u_{b, j}+\gamma_{1} u_{1, j}+\gamma_{2} u_{2, j}+\gamma_{3} u_{3, j}+\cdots .
\end{aligned}
$$

The coefficients $\beta$ and $\gamma$ are to be determined from order and symmetry requirements. Requiring skew-symmetry in case of $\overline{\bar{u}}_{b, j}=0$ leads to

$$
\begin{array}{r}
\delta_{0}-\delta_{1}+\beta_{1}=0, \\
-\delta_{2}-\beta_{0}+\beta_{2}=0, \\
\delta_{2}-\beta_{1}=0,
\end{array}
$$




$$
\begin{aligned}
& \delta_{1}=\beta_{2}=\frac{1}{2}, \\
& \delta_{3}=\beta_{3}=0 .
\end{aligned}
$$

Requiring a consistent (first-order) interpolation gives the conditions $\delta_{0}+\delta_{1}+\delta_{2}=1$ and $\beta_{0}+\beta_{1}+\beta_{2}=1$. The solution to these equations leads to interpolations (B.1)-(B.2). The resulting coefficients also satisfy the conditions for second order interpolation, but not higher order conditions. Skew-symmetry and higher order are therefore conflicting requirements. Higher-order treatment of boundary conditions, as in [14] for instance, violates the skew-symmetry of the convective operator.

An important starting point in our analysis is that (skew-)symmetry is defined in terms of the standard inner product, Eq. (26). When generalizing the inner product higher order can be combined with symmetry properties, see e.g. [20,12] for hyperbolic and parabolic problems, respectively. Perhaps also for the incompressible Navier-Stokes equations such an adapted inner product can be found. However, the pressure will then contribute to the energy equation. This is a subject of further research.

\subsection{Summary of new boundary conditions}

In Table 1 we summarize the old and new boundary conditions for the fourth order scheme needed for the discretization of the convective terms and the divergence-free constraint in order to make the convective terms energy-conserving. The boundary conditions for the diffusive terms are unaltered and are of Dirichlet type. The old boundary conditions (110) form a rather unclear mix of Dirichlet and Neumann conditions for both $u$ - and $v$-component. The new boundary conditions (111) feature Dirichlet conditions for the $u$-component, whereas Neumann type conditions are prescribed for the $v$-component. Furthermore, our new discretization of the $v$-component near the wall does not require the prescription of $v-2, j$.

Some remarks on the 'multi-valuedness' of the $v$-component near a solid wall (Neumann for convective terms, Dirichlet for diffusive terms) are in place. First we note that for a one-dimensional problem, e.g. the convection-diffusion equation (see also Section 4), the boundary conditions for both convective and diffusive terms are the same, and are simply of Dirichlet (no-slip) type. In two dimensions this is still true for the $u$-component at a vertical boundary, but for the $v$-component it only holds for the diffusive terms. The discrepancy appears when the mass conservation constraint appears, in fact upon requiring mass conservation of a ghost volume, Eq. (39). A ghost cell need not have a physical interpretation, it is just a way to implement the boundary conditions. Here, the velocity in the ghost cell becomes multi-valued. If the boundary condition is added to the convective operator, the incompressibility constraint is to be taken into account, since otherwise the skew-symmetry and energy conserving property is lost. The diffusive operator, however, does not require the incompressibility constraint to be symmetric. In other words, the boundary treatment of the convective term is more restricted (by incompressibility). Applying that treatment to the diffusive term would reduce the accuracy of the diffusive part of the discretization. Therefore, we discretize the boundary conditions for the convective and diffusive terms in a different manner. This leads to multi-valued ghost velocities.

Secondly, in literature it is not uncommon to have discretization methods that have different ghost values for convection and diffusion discretizations. For example, the high-order summation-by-part discretizations in adapted inner products described by Mattson and Nordström [12] effectively have different boundary conditions for the convective and diffusive terms, which becomes clear when rewriting their finite difference stencil in terms of ghost values.

Table 1

Old and new boundary conditions for fourth order discretization of divergence constraint and convective terms.

\begin{tabular}{ll}
\hline Old [26] & New \\
\hline$u_{-1, j}=2 u_{b, j}-u_{1, j}$ & $u_{-1, j}=2 u_{b, j}-u_{1, j}$ \\
$u_{-2, j}=u_{2, j}$ & $u_{-2, j}=2 u_{b, j}-u_{2, j}$ \\
$\bar{u}_{-1, j}=\bar{u}_{1, j}$ & $\bar{u}_{-1, j}=2 \bar{u}_{b, j}-\bar{u}_{1, j}$ \\
$\overline{\bar{u}}_{-1, j}=\overline{\bar{u}}_{1, j}$ & $\overline{\bar{u}}_{-1, j}=2 \overline{\bar{u}}_{b, j}-\overline{\bar{u}}_{1, j}$ \\
$\overline{\bar{u}}_{-2, j}=\overline{\bar{u}}_{2, j}$ & $\overline{\bar{u}}_{-2, j}=2 \overline{\bar{u}}_{b, j}-\overline{\bar{u}}_{2, j}$ \\
$v_{0, j}=2 v_{b, j}-v_{1, j}$ & $v_{0, j}=v_{1, j}$ \\
$\bar{v}_{0, j}=2 \bar{v}_{b, j}-\bar{v}_{1, j}$ & $\bar{v}_{0, j}=\bar{v}_{1, j}$ \\
$\overline{\bar{v}}_{0, j}=2 \overline{\bar{v}}_{b, j}-\overline{\bar{v}}_{1, j}$ & $\overline{\bar{v}}_{0, j}=\bar{v}_{1, j}$ \\
$v_{-2, j}=v_{3, j}$ & \\
\hline
\end{tabular}




\subsection{Pressure}

Like in the continuous case, in a discrete setting the pressure Poisson equation is formed by differentiating the divergence-free constraint (in time) and substituting the momentum equations:

$$
M \Omega^{-1} G p=M \Omega^{-1}(-C(c) u+D u)=M N(u)
$$

The boundary conditions are 'built' into the operators $M$ and $G$; see the discussion at the end of Section 3.4. To obtain the effective boundary conditions for the pressure, we compare the Laplace operator at the boundary to the Laplace operator at an inner point, and subtract these two [22,4]. For the second order method it is known that this gives a boundary condition which is the discrete equivalent of Eq. (23). In Appendix $C$ we detail such an analysis for the fourth order method. The resulting effective boundary conditions at $i=1, i=2$ and $i=3$ are

$$
\begin{aligned}
& \frac{1}{\Delta x}\left(\frac{1}{8}\left(p_{3, j}-p_{-2}\right)-\frac{27}{4}\left(p_{2, j}-p_{-1, j}\right)+\frac{783}{8}\left(p_{1, j}-p_{0, j}\right)\right)=\alpha_{1} N_{b, j}^{u}-\alpha_{2}\left(N_{-1, j}^{u}+N_{1, j}^{u}\right)+\left(-\alpha_{1}+2 \alpha_{2}\right) \dot{u}_{b, j} \\
& \frac{1}{\Delta x}\left(\frac{1}{8}\left(p_{2, j}-p_{-1, j}\right)-\frac{27}{4}\left(p_{1, j}-p_{0, j}\right)\right)=-\alpha_{2} N_{b, j}^{u}+\alpha_{2} \dot{u}_{b, j} \\
& \frac{1}{\Delta x} \frac{1}{8}\left(p_{1, j}-p_{0, j}\right)=0 .
\end{aligned}
$$

The effective pressure boundary conditions at the three points $i=1,2$, and 3 are not consistent approximations to the continuous boundary conditions (23). This appears to be in line with a remark made by Sani et al. [19], who state 'Whenever the discrete PPE is generated from a consistent (but low order) discretization of the Navier-Stokes equations, the resulting PPE will always converge to (23)'. On average the pressure boundary conditions are still correct. This follows by summing the effective boundary conditions for $i=1, i=2$ and $i=3$, leading to

$$
\begin{aligned}
\frac{1}{\Delta x}\left(\frac{1}{8}\left(p_{3, j}-p_{-2}\right)-\frac{53}{8}\left(p_{2, j}-p_{-1, j}\right)+\right. & \left.\frac{365}{4}\left(p_{1, j}-p_{0, j}\right)\right) \\
& =\left(\alpha_{1}-\alpha_{2}\right) N_{b, j}^{u}-\alpha_{2}\left(N_{1, j}^{u}+N_{-1, j}^{u}\right)+\left(-\alpha_{1}+3 \alpha_{2}\right) \dot{u}_{b, j}
\end{aligned}
$$

Expanding the left side in Taylor expansions around the boundary gives

$$
\frac{1}{\Delta x}\left(\frac{1}{8}\left(p_{3, j}-p_{-2}\right)-\frac{53}{8}\left(p_{2, j}-p_{-1, j}\right)+\frac{365}{4}\left(p_{1, j}-p_{0, j}\right)\right)=72\left(p_{x}\right)_{b}-3 \Delta x^{2} p_{x x x}+\mathcal{O}\left(\Delta x^{4}\right)
$$

Similarly, expansion of the right side gives

$$
\left(\alpha_{1}-\alpha_{2}\right) N_{b, j}^{u}-\alpha_{2}\left(N_{1, j}^{u}+N_{-1, j}^{u}\right)+\left(-\alpha_{1}+3 \alpha_{2}\right) \dot{u}_{b, j}=-72 \dot{u}_{b, j}+72 N_{b, j}^{u}-3 \Delta x^{2}\left(N_{x x}^{u}\right)_{b, j}+\mathcal{O}\left(\Delta x^{4}\right) .
$$

Upon division by the effective (pressure) volume size we find that the fourth order method leads to a consistent effective boundary condition for the pressure:

$$
\left(p_{x}\right)_{b}=N_{b, j}^{u}-\dot{u}_{b, j}+\mathcal{O}\left(\Delta x^{2}\right)
$$

which is the discrete approximation to Eq. (23).

\subsection{Temporal discretization}

The temporal discretization has not been considered so far. Conservation of mass is not an evolution equation but a constraint which should be satisfied independent of the particular time integration method. The requirement on the temporal discretization that results from conservation of momentum is consistency. All standard multi-step (like BDF, Adams) and multi-stage (Runge-Kutta) methods are consistent. Conservation of kinetic energy, a quadratic quantity, poses strict conditions on the time integration method. Ham et al. [5] employed the implicit midpoint method to obtain energy conservation in space and time. This second order method is an example of a Runge-Kutta method based on Gauss quadrature, which conserves energy in time. They are discussed in more detail in [18]. We will use the implicit midpoint method to demonstrate energy conservation in both time and space in the numerical simulations that will be presented later. 


\section{Order of accuracy analysis}

In this section we investigate the effect of symmetry-preserving boundary conditions on local and global order behavior We consider the linear convection-diffusion equation as a special case of the Navier-Stokes equations (2) in one dimension:

$$
c \frac{\mathrm{d} \hat{u}}{\mathrm{~d} x}=v \frac{\mathrm{d}^{2} \hat{u}}{\mathrm{~d} x^{2}}, \quad 0 \leqslant x \leqslant 1, c<0, v>0,
$$

where $c$ is the convecting velocity, $\hat{u}$ the convected velocity and $v$ the viscosity coefficient. To study the effect of no-slip conditions, Eq. (120) is supplemented with Dirichlet boundary conditions:

$$
\hat{u}(0)=\hat{u}_{b}=0, \quad \hat{u}(1)=1 .
$$

In operator notation equation (120) can be concisely written as

$$
\hat{L} \hat{u}=\hat{f},
$$

where

$$
\hat{L} \equiv c \frac{\mathrm{d}}{\mathrm{d} x}-v \frac{\mathrm{d}^{2}}{\mathrm{~d} x^{2}}, \quad \hat{f}=0 .
$$

For small values of $v$ a boundary layer develops at $x=0$. Defining $\varepsilon=v / c(\varepsilon<0)$, the exact solution to Eq. (120) is given by

$$
\hat{u}(x)=\frac{e^{x / \varepsilon}-1}{e^{1 / \varepsilon}-1} .
$$

\subsection{Discretization}

The discretization of the convective term, Eq. (A.11), is restricted to $1 \mathrm{D}$ by taking $c=c_{x}=$ constant and $c_{y}=0$ :

$$
(C u)_{i}=\alpha_{1}\left(\frac{u_{i+1}+u_{i}}{2}-\frac{u_{i}+u_{i-1}}{2}\right)-\left(\frac{u_{i+3}+u_{i}}{2}-\frac{u_{i}+u_{i-3}}{2}\right),
$$

where $\alpha=3^{3}=27$. Without loss of generality we consider the discretization at the left boundary (where the boundary layer is located). Following Section 3.6, these boundary conditions are given by Eq. (111):

$$
u_{0}=u_{b}, \quad u_{-1}=2 u_{b}-u_{1}, \quad u_{-2}=2 u_{b}-u_{2} .
$$

Restricting the convective discretization for the coarse grid, Eq. (B.3), to one dimension $(\overline{\bar{u}}=c, \overline{\bar{v}}=0)$ leads to

$$
C_{3}=\left[\begin{array}{ccccc}
-\frac{1}{2} & 0 & 0 & \frac{1}{2} & \\
\cline { 2 - 5 }-1 & 0 & \frac{1}{2} & 0 & \ddots \\
-1 & \frac{1}{2} & 0 & 0 & \\
-\frac{1}{2} & 0 & 0 & \ddots & \\
& \ddots & & &
\end{array}\right] .
$$

The lower right part of the matrix is the discretization of the interior points, which is used in practical implementation. We note that there is a fundamental difference between the 2D non-linear case and the 1D linear case. In the 2D non-linear case $c=u$, and $C_{3}$ is skew-symmetric for $u_{b}=0$. A homogeneous Dirichlet condition for $u$ implies a homogeneous Dirichlet condition for $c$. In the $1 \mathrm{D}$ linear case, $c=$ constant is prescribed, which is different from the boundary condition for $u$. The convective matrix is therefore not skew-symmetric.

The resulting scheme is written as follows:

$$
L u=f,
$$

where

$$
L=c C-v D,
$$

and

$$
f=-c f^{c}+v f^{d} .
$$


The upper left corner of the convective part reads (considering only interior points):

$$
C=\alpha_{1} C_{1}-C_{3}=\frac{1}{2}\left[\begin{array}{cccccc}
0 & \alpha_{1}-1 & 0 & -1 & & \\
-\alpha_{1}-1 & 0 & \alpha_{1} & 0 & -1 & \\
0 & -\alpha_{1} & 0 & \alpha_{1} & 0 & \ddots \\
1 & 0 & -\alpha_{1} & 0 & \alpha_{1} & \\
& \ddots & & \ddots & & \ddots
\end{array}\right], \quad f^{c}=\frac{u_{b}}{2}\left[\begin{array}{c}
-\left(\alpha_{1}-2\right) \\
2 \\
1 \\
0 \\
\vdots
\end{array}\right] .
$$

The diffusive part reads

$$
\begin{aligned}
& D=\frac{1}{24 h}\left[\begin{array}{cccccc}
-2 \alpha_{1}^{2}+2 \alpha_{1}-2 & \alpha_{1}^{2}+2 \alpha_{1}-1 & -2 \alpha_{1} & 1 & & \\
\alpha_{1}^{2}+2 \alpha_{1}-1 & -2 \alpha_{1}^{2}-2 & \alpha_{1}^{2}+2 \alpha_{1} & -2 \alpha_{1} & 1 & \\
-2 \alpha_{1} & \alpha_{1}^{2}+2 \alpha_{1} & -2 \alpha_{1}^{2}-2 & \alpha_{1}^{2}+2 \alpha_{1} & -2 \alpha_{1} & \ddots \\
1 & -2 \alpha_{1} & \alpha_{1}^{2}+2 \alpha_{1} & -2 \alpha_{1}^{2}-2 & \alpha_{1}^{2}+2 \alpha_{1} & \\
& \ddots & & \ddots & & \ddots
\end{array}\right], \\
& f^{d}=\frac{u_{b}}{24 h}\left[\begin{array}{c}
\alpha_{1}^{2}-2 \alpha_{1}+2 \\
-2 \alpha_{1}+2 \\
1 \\
0 \\
\vdots
\end{array}\right] \text {. }
\end{aligned}
$$

The discretization at the right boundary is done in a similar fashion.

\subsection{Local truncation error}

In order to study the error behavior we find expressions for the local truncation error and relate it to the global truncation error. The local truncation error is defined as

$$
\tau \equiv L \hat{u}-f .
$$

Note that strictly speaking a restriction operator is necessary to map the continuous function $\hat{u}$ to the space of discrete functions in order to be able to apply $L$; in this case the restriction operator is a simple injection: $\hat{u}_{i}=\hat{u}\left(x_{i}\right)$. The global error is defined as

$$
e \equiv \hat{u}-u,
$$

which is related to $\tau$ as

$$
L e=\tau .
$$

Note that the global error is, like the local error, a quantity defined in each finite volume. The notion of 'global' is due to the fact that $L^{-1}$ is a nonlocal operator, which distributes the local error $\tau$.

First we discuss the local truncation error of the convective terms, on a uniform grid. We Taylor expand the exact solution $\hat{u}$ around a point $x_{i}$ :

$$
\hat{u}\left(x_{i}+h\right)=\hat{u}\left(x_{i}\right)+h \hat{u}_{i}^{(1)}+\frac{1}{2} h^{2} \hat{u}_{i}^{(2)}+\mathcal{O}\left(h^{3}\right),
$$

where $\hat{u}_{i}^{(n)}=\left(\frac{\mathrm{d}^{n} \hat{u}}{\mathrm{~d} x^{n}}\right)_{i}$, and we apply operator $C$ :

$$
\tau_{i}^{c}=-\frac{9}{5} h^{5} \hat{u}_{i}^{(5)}+\mathcal{O}\left(h^{7}\right) .
$$

The truncation error looks fifth order instead of fourth order due to a factor $h$ hidden in $C$ (due to the integration over a finite volume). To find $\tau$ at $i=1$ and 2 , where the stencil changes due to boundary conditions, we also employ a Taylor expansion for $\hat{u}_{b}$ :

$$
\hat{u}_{b}=\hat{u}_{1}-h \hat{u}_{1}^{(1)}+\frac{1}{2} h^{2} \hat{u}_{1}^{(2)}+\mathcal{O}\left(h^{3}\right)
$$

This leads to the following truncation errors: 


$$
\begin{aligned}
\tau_{1}^{c} & =-2 h^{2} \hat{u}_{1}^{(2)}+\mathcal{O}\left(h^{3}\right), \\
\tau_{2}^{c} & =-\frac{1}{2} h^{2} \hat{u}_{2}^{(2)}+\mathcal{O}\left(h^{3}\right) .
\end{aligned}
$$

It turns out that the local truncation error of the convective discretization at $i=1$ and $i=2$ is limited to first order.

The same procedure is followed to analyze the truncation error of the diffusive terms:

$$
\begin{aligned}
\tau_{i}^{d} & =-\frac{9}{40} h^{5} \hat{u}_{i}^{(6)}+\mathcal{O}\left(h^{7}\right), \\
\tau_{1}^{d} & =\frac{25}{12} h \hat{u}_{1}^{(2)}-\frac{25}{12} h^{2} \hat{u}_{1}^{(3)}+\mathcal{O}\left(h^{3}\right), \\
\tau_{2}^{d} & =-\frac{1}{24} h \hat{u}_{2}^{(2)}+\frac{1}{12} h^{2} \hat{u}_{2}^{(3)}+\mathcal{O}\left(h^{3}\right) .
\end{aligned}
$$

The diffusive discretization is inconsistent at both $i=1$ and $i=2$.

\subsection{Global truncation error}

Although the discretization at the boundaries is inconsistent, it is possible to have convergence in the global error. Several techniques exist to study the relation between the local and global error, see for example $[11,7,15]$. We use the approach outlined in Wesseling [27]. Recall the relation between the global and local truncation error, Eq. (136):

$$
L e=\tau .
$$

We assume that $L$ is a monotone operator, i.e.

$$
L v \geqslant 0 \text { implies } v \geqslant 0 .
$$

Equivalently one can say that $L$ is monotone if and only if $L^{-1} \geqslant 0 .{ }^{2}$ If we can construct a function $E$, a so-called barrier function, such that ${ }^{3}$

$$
L E \geqslant|\tau|,
$$

then, subtracting this equation from (145), we obtain

$$
L(e-E) \leqslant 0 .
$$

Since $L$ is assumed monotone, this means that

$$
e-E \leqslant 0 \rightarrow e \leqslant E .
$$

The art is to construct a barrier function $E$ of a certain order $p, E \sim h^{p}$, such that (147) holds. Eq. (149) then proves that the global error $e$ is also of order $p$. Since any sufficiently differentiable continuous function can be expressed as a polynomial expansion, we take $E=h^{p} \psi(x)$ with

$$
\psi(x)=a_{0}+a_{1} x+a_{2} x^{2}+a_{3} x^{3}+a_{4} x^{4} .
$$

It is possible to include more terms in this expansion, but it will turn out that this is not necessary.

Note that proving monotonicity of $L$ for the fourth-order discretization is not trivial. Normally, monotonicity can be proven by showing that $L$ is positive. ${ }^{4}$ For second-order central discretizations positivity is proven when the mesh Péclet number $h / v$ is smaller than 2. For the fourth-order scheme this approach fails, because $L$ can never be positive, as can be observed by inspecting the signs of the diagonals of $L$. However, a non-positive operator can still be monotone (see for example $[8,1]$ ). We have not yet found a sufficient condition for monotonicity of the fourth-order scheme, but it suffices to say that we have found, by explicitly computing $L^{-1}$, that $L$ is monotone if $h / v$ is small enough.

\footnotetext{
$2 a \geqslant 0$ means that $a_{i} \geqslant 0 \forall i ; A \geqslant 0$ means that $A_{i j} \geqslant 0 \forall i, j$.

$3|a|$ is the grid function with value $\left|a_{i}\right|$.

4 Positivity implies monotonicity, but not vice versa.
} 


\subsubsection{Interior}

In a general interior point $i$ we can write for $L E$ :

$$
L\left(h^{p} \psi(x)\right)_{i}=24 h^{p+1}\left(c\left(4 a_{4} x_{i}^{3}+3 a_{3} x_{i}^{2}+2 a_{2} x_{i}+a_{1}\right)-v\left(12 a_{4} x_{i}^{2}+6 a_{3} x_{i}+2 a_{2}\right)\right) .
$$

We recognize the exact first and second derivatives of $\psi(x)$. This is because $\tau_{i}^{c}$ and $\tau_{i}^{d}$ only contain derivatives of fifth order and higher: $L$ can differentiate a fourth-order polynomial exactly. Of course one can take more terms in expansion (150); these will not be exactly differentiated. Since $\tau_{i}$ is

$$
\tau_{i}=c \tau_{i}^{c}-v \tau_{i}^{d}=-c \frac{9}{5} h^{5} \hat{u}_{i}^{(5)}+v \frac{9}{40} h^{5} \hat{u}_{i}^{(6)}+\mathcal{O}\left(h^{7}\right),
$$

we find that $L E-|\tau|$ can be written as

$$
\begin{aligned}
L E_{i}-\left|\tau_{i}\right|= & 24 h^{p+1}\left(c\left(4 a_{4} x_{i}^{3}+3 a_{3} x_{i}^{2}+2 a_{2} x_{i}+a_{1}\right)-v\left(12 a_{4} x_{i}^{2}+6 a_{3} x_{i}+2 a_{2}\right)\right) \\
& -h^{5}\left|-c \frac{9}{5} \hat{u}_{i}^{(5)}+v \frac{9}{40} \hat{u}_{i}^{(6)}\right|+\mathcal{O}\left(h^{7}\right) .
\end{aligned}
$$

For $p \leqslant 4$ it is possible to construct the coefficients $a_{n}$ such that $L E-|\tau| \geqslant 0$. This indicates that a fourth-order accurate local truncation error leads to a fourth-order accurate global error, as expected.

\subsubsection{Boundary, $i=1$}

The analysis is now repeated for the boundary points. At $i=1\left(x_{1}=h\right)$ the local error is

$$
\tau_{1}=c \tau_{1}^{c}-v \tau_{1}^{d}=-v \frac{25}{12} h \hat{u}_{1}^{(2)}-c 2 h^{2} \hat{u}_{1}^{(2)}+v \frac{25}{12} h^{2} \hat{u}_{1}^{(3)}+\mathcal{O}\left(h^{3}\right)
$$

so we obtain

$$
L\left(h^{p} \psi(x)\right)_{1}-\left|\tau_{1}\right|=h^{p}\left(c \frac{27}{2} a_{0}+v \frac{677}{24 h} a_{0}+\mathcal{O}(h)\right)-h\left|-v \frac{25}{12} \hat{u}_{1}^{(2)}+\mathcal{O}(h)\right| .
$$

To make sure that the order of the first term does not exceed the order of the truncation error (for $h \rightarrow 0$ ), we require $p \leqslant 2$. For example, in case $p=2$, the leading order term condition is

$$
h\left(\frac{677}{24} v a_{0}-\left|-v \frac{25}{12} \hat{u}_{1}^{(2)}\right|\right) \geqslant 0 .
$$

This means that with a sufficiently large positive value of $a_{0}$ we have a barrier function that is always larger than $|\tau|$ for sufficiently small $h$. Only the first term of the polynomial expansion (150) is of importance here; increasing the polynomial order will not change Eq. (156).

\subsubsection{Boundary, $i=2$}

At $i=2\left(x_{2}=2 h\right)$, we have

$$
\tau_{2}=c \tau_{2}^{c}-v \tau_{2}^{d}=v \frac{1}{24} h \hat{u}_{2}^{(2)}-c \frac{1}{2} h^{2} \hat{u}_{2}^{(2)}-v \frac{1}{12} h^{2} \hat{u}_{2}^{(3)}+\mathcal{O}\left(h^{3}\right),
$$

and we obtain

$$
L\left(h^{p} \psi(x)\right)_{2}-\left|\tau_{2}\right|=h^{p}\left(-c a_{0}-v \frac{13}{6 h} a_{0}\right)-h\left|v \frac{1}{24} \hat{u}_{2}^{(2)}+\mathcal{O}(h)\right| .
$$

This indicates again that the maximum order of the global error is $p=2$ if the following condition can be fulfilled:

$$
h\left(-\frac{13}{6} v a_{0}-\left|v \frac{1}{24} \hat{u}_{2}^{(2)}+\mathcal{O}(h)\right|\right) \geqslant 0 .
$$

However, this requires $a_{0}<0$, which contradicts the requirement for $i=1$. For the current boundary layer problem we can escape from this by noting that $\tau_{1}>0, \tau_{2}<0$ and $\left|\tau_{1}\right|>\left|\tau_{2}\right|$. This means that the absolute value signs are too restrictive; a range of positive values of $a_{0}$ exists for which both (156) and (159) are satisfied.

Summarizing the results for $i=1$ and $i=2$, we find that no polynomial barrier function exists with which we can prove a convergence rate higher than 2 . We expect that this result holds for other barrier functions, given that any sufficiently differentiable function on the domain $x \in[0,1]$ can be expressed as a polynomial. Consequently, the global error of the fourth order scheme is limited to second order due to boundary conditions. 


\section{Numerical experiments}

A number of numerical experiments are carried out to support the theoretical results from Sections 3 and 4 . First, we study the order of accuracy of the proposed boundary conditions for a one-dimensional convection-diffusion equation (Section 5.1) and a two-dimensional lid-driven cavity (Section 5.2), and how fourth order accuracy can be obtained on properly designed non-uniform grids. Secondly, the energy conservation properties of the new boundary conditions are demonstrated in Section 5.3 by considering inviscid flow in a cavity with non-zero tangential boundary conditions. Lastly, in Section 5.4 we consider three-dimensional turbulent channel flow for which we show that the new boundary conditions improve both the velocity and pressure field near the wall, compared to the old conditions.

\subsection{D convection-diffusion equation}

\subsubsection{Uniform grid}

First we verify the fourth-order scheme by using 'exact' boundary conditions, i.e. we substitute the exact solution (124) for the ghost points, like in Veldman [23]. For $v=1 / 100$ and $h=1 / 200(|h / \varepsilon|=1 / 2)$ the discrete and exact solution are shown in Fig. 5(a). The global truncation error $e=\hat{u}-u$ and local truncation error, obtained from $\tau=L e$, are shown in Fig. 5(b). The leading error term of the theoretical local truncation error, Eq. (152), is found by using the exact solution, Eq. (124):

$$
\tau_{i}=\left(-\frac{9}{5}+\frac{9}{40}\right)\left(\frac{h}{\varepsilon}\right)^{5} \frac{e^{x_{i} / \varepsilon}}{e^{1 / \varepsilon}-1},
$$

and is also shown in Fig. 5(b). Because Eq. (160) contains only the leading error term, there is a small deviation between the two local truncation errors. The local truncation error always attains its maximum (in absolute sense) at $i=1$, because $e^{x / \varepsilon}$ is largest here (note $\varepsilon<0$ ). The global error, on the other hand, does not necessarily attain its maximum value in the first grid point: this depends on $L^{-1}$. The structure of $L^{-1}$ is shown in Fig. 5(c); all entries are $\geqslant 0$, so $L$ is indeed monotone in this case. It can be seen that $L^{-1}$ distributes the local error $\tau$ over the entire domain in a particular way. For instance, $e_{1}$ is influenced by $\tau$ in the entire domain, whereas $e_{N}$ is mainly influenced by $\tau$ at the right side of the domain. This is because the 'flow' is convection-dominated and information travels from right to left $(c<0)$.

A plot of $\|e\|_{\infty}$ as a function of $h$ displays a clear fourth-order convergence, see Fig. 5(d). The local error $\|\tau\|_{\infty}$ shows fifth-order convergence, as expected from (160).

Now we turn to the results for scheme (128)-(133) where the boundary conditions are chosen such that symmetry properties of the discrete operators are retained. Fig. 6(a) shows that the behavior of the local truncation error near the boundary is much more irregular, as expected. As predicted by Eqs. (154) and (157), the convergence of the local error at the boundary is first order for sufficiently small $h$. The global error shows second-order convergence, confirming the barrier function analysis from Section 4.3. In the $L_{2}$ norm the convergence of local and global error is very similar. Note that numerical experiments showed that (at $v=1 / 100$ ) $L$ is monotone for $h<1 / 60$, approximately.

The 'kink' in the global and local error around $h=10^{-2}$ is due to the fact that the local truncation errors in the convective and diffusive terms almost cancel each other at $i=1$. This can also be seen from Fig. 6(c), which shows that the position of maximum $\tau$ shifts from $i=1$ to $i=2$.

Considering that both theoretical arguments and numerical experiments show that the fourth-order symmetry-preserving method is only second-order accurate for boundary layer problems with Dirichlet boundary conditions, one might wonder if it is useful to apply a fourth-order method anyway. We therefore compare the results presented above with the secondorder method, which does not require boundary conditions for $u_{-1}$ and $u_{-2}$. The global error behavior of the second- and fourth-order schemes is shown in Fig. 6(d). It is observed that for most meshes, except the very coarse ones, the secondorder method is more accurate than the fourth-order method. This is due to the fact that in this test case the largest gradients in the solution are near the boundary, where the local truncation error has unfavorable properties. On uniform grids with boundary-layer like problems the fourth-order method is not preferred over the second-order method.

We will continue to study the effect of the boundary conditions and the difference between the second and fourth order scheme by considering non-uniform grids. Such grids are more suitable to handle boundary-layer like problems than uniform grids.

\subsubsection{Non-uniform grid}

In practice, thin boundary layers should be calculated by employing non-uniform grids. In this section we investigate if the conclusions for uniform grids carry over to non-uniform grids. First we investigate an exponential grid, i.e. a grid where each cell size is a constant factor times the neighboring grid cell size. The grid is written as a mapping of a uniform grid,

$$
x(\xi)=\left(1-s^{\xi}\right) /(1-s),
$$

where $\xi$ is uniformly distributed over $[0,1]$, and $s$ is the stretch factor. The stretch factor is determined by choosing a refinement region $\delta$ such that in both $[0, \delta]$ and $[\delta, 1] N / 2$ volumes are located. The stretch factor stays constant during mesh refinement. Defining the boundary layer edge as the point where $\hat{u}=p \hat{u}(1)$, we find for sufficiently small $\varepsilon$ : 


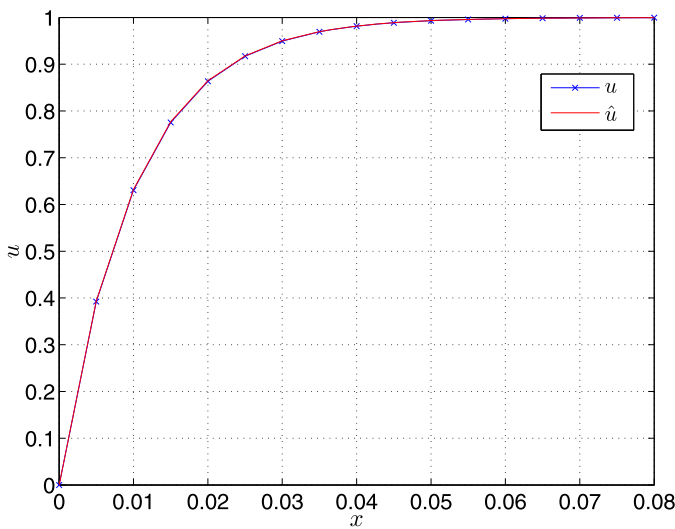

(a) Discrete and exact solution, $h=1 / 200$.

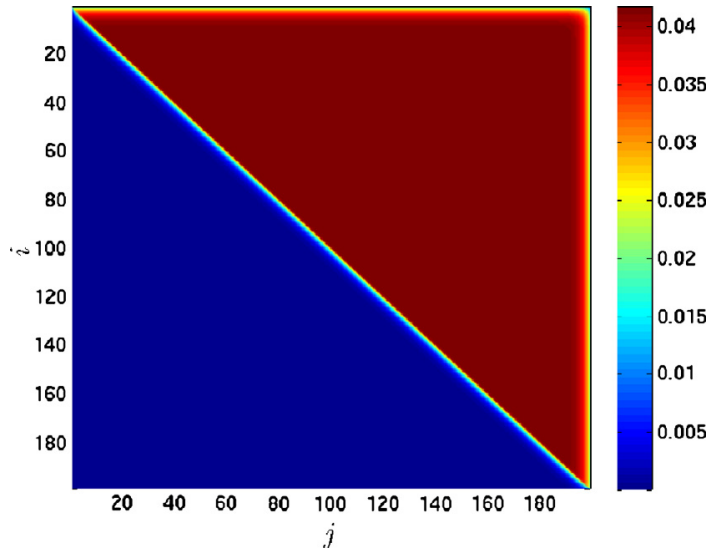

(c) Structure of $L^{-1}, h=1 / 200$.

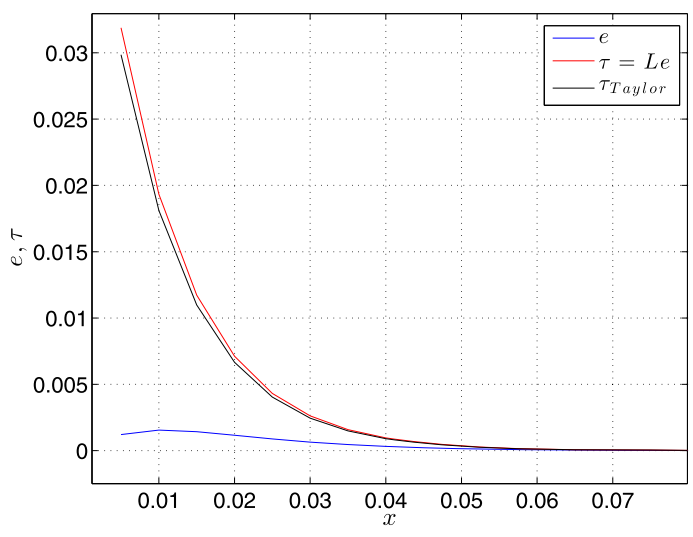

(b) Global and local truncation error, $h=1 / 200$.

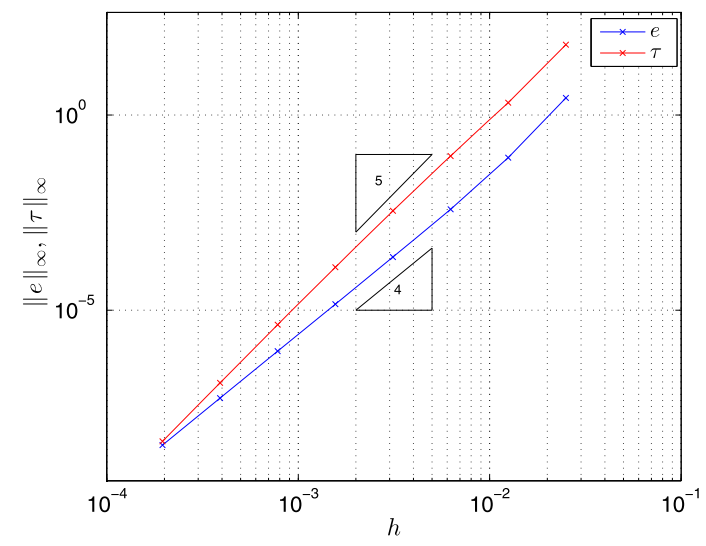

(d) Convergence of global error.

Fig. 5. Solution and error behavior for uniform grid, exact boundary conditions, $v=1 / 100$.

$$
\delta_{p}=\varepsilon \ln (1-p) .
$$

For example, if the boundary layer edge is defined at the point where $\hat{u}=0.9$, then $\delta_{0.9} \approx 2.3 \cdot 10^{-2}(\varepsilon=-1 / 100)$. Looking at the convergence and position of the local and global error for $\delta_{0.9}$ (Figs. 7(a) and 7(b)) it can be seen that the refinement region can make sure that the position of maximum global error is not at the boundary; $u(\xi)$ does not have its largest gradient at the boundary. However, for sufficiently small $h$ the global error moves to the boundary, and the slope changes to second order $\left(h \approx 10^{-2}\right)$, like in the uniform case. The position of the kink, $h_{k}$, is mainly determined by $\delta$ : the smaller $\delta$, the smaller the value of $h_{k}$. If the refinement zone is chosen properly, the error will exhibit a region of fourth order convergence. For these non-uniform grids, the fourth-order scheme is more accurate than its second-order counterpart.

An important question that remains is: can we design the mesh such that the global error keeps its fourth order behavior upon mesh refinement? We concluded in Section 4 that the local truncation error near boundaries is $\mathcal{O}(1)$, resulting in a global error of $\mathcal{O}\left(h^{2}\right)$. If we construct the mesh near the boundary such that $h_{b n d}=\mathcal{O}\left(h^{2}\right)$ (where $h=1 / N$ ), then one might expect heuristically a contribution to the global error of $\mathcal{O}\left(h^{4}\right)$. Such a mesh can be constructed by making the stretch factor $h$-dependent. The smallest finite volume is given by

$$
h_{1}=\frac{1-s^{h}}{1-s}=-\frac{\ln (s)}{1-s} h+\mathcal{O}\left(h^{2}\right) .
$$

With $s \sim 1 / h$ we obtain $h_{1}=\mathcal{O}\left(h^{2}\right)$. In Fig. 8 we show the convergence of the global error for the case $s=10 / h$. It is clearly seen that the error now converges with $\mathcal{O}\left(h^{4}\right)$, for all mesh sizes. The fourth order method is now much more accurate than the second order method.

To summarize, the fourth order scheme attains fourth order convergence on properly chosen non-uniform meshes. These meshes should be designed such that the global error is not at the boundary but in the interior of the domain: the smallest cells near the boundary should scale with $\mathcal{O}\left(h^{2}\right)$ upon mesh refinement. Furthermore, the mesh should be 'smooth' enough to exhibit fourth order behavior. Recalling that the relation between the derivative on a uniform and non-uniform grid is given by 


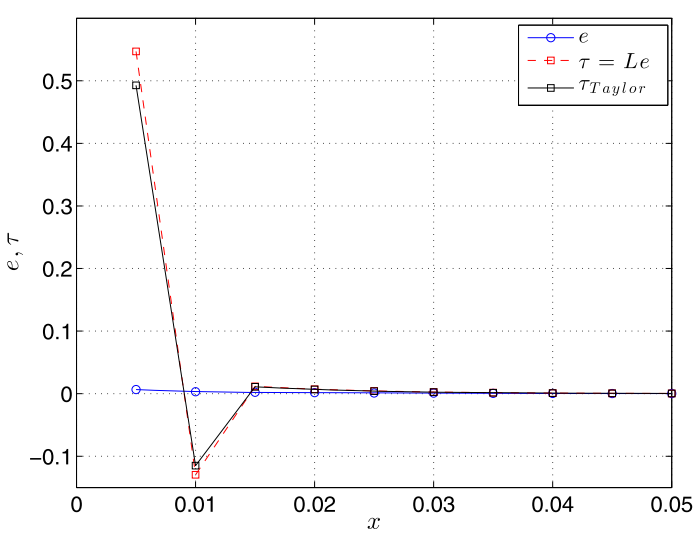

(a) Global and local truncation error, $h=1 / 200$.

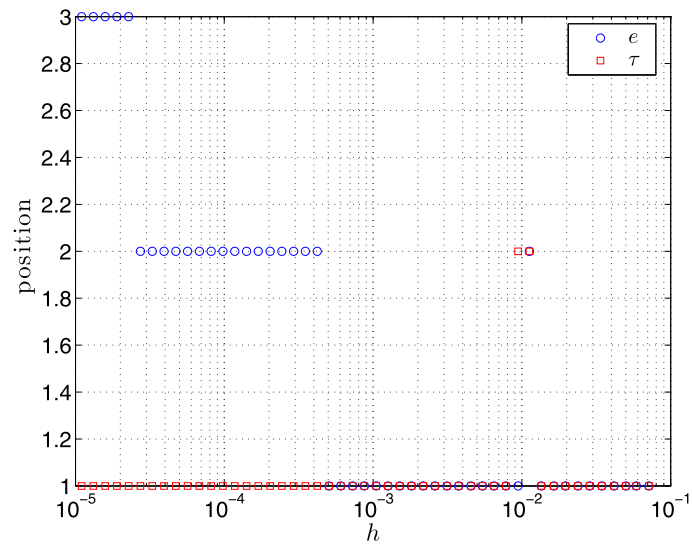

(c) Position of maximum global and local error.

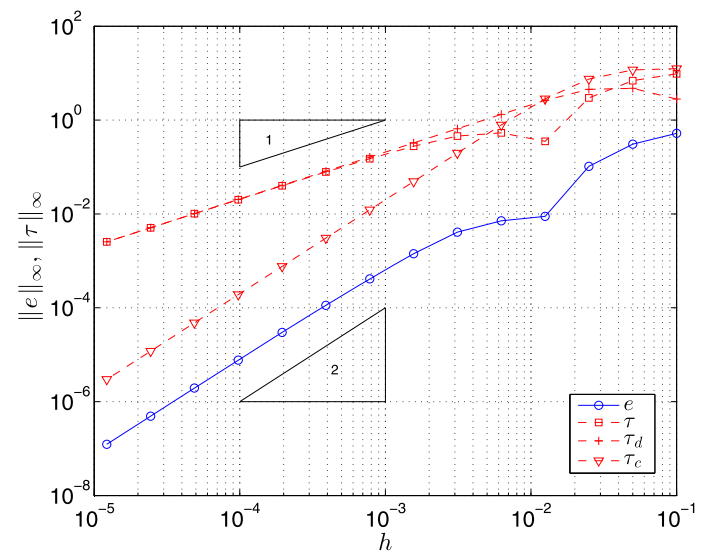

(b) Convergence of global and local error.

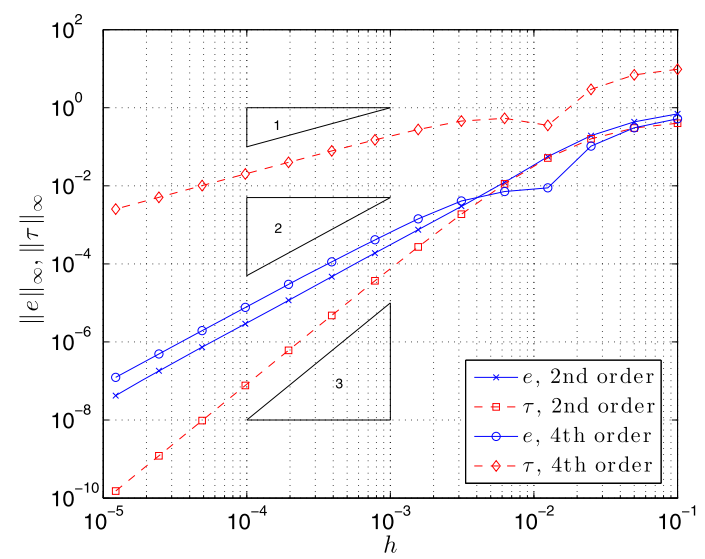

(d) Comparison with second-order scheme.

Fig. 6. Error behavior for uniform grid, symmetry-preserving boundary conditions, $v=1 / 100$.

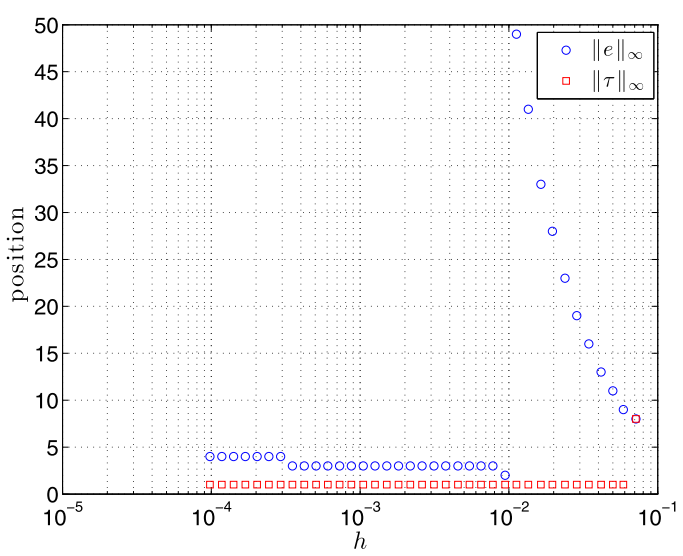

(a) Position of maximum global and local error, $\delta_{0.9}$.

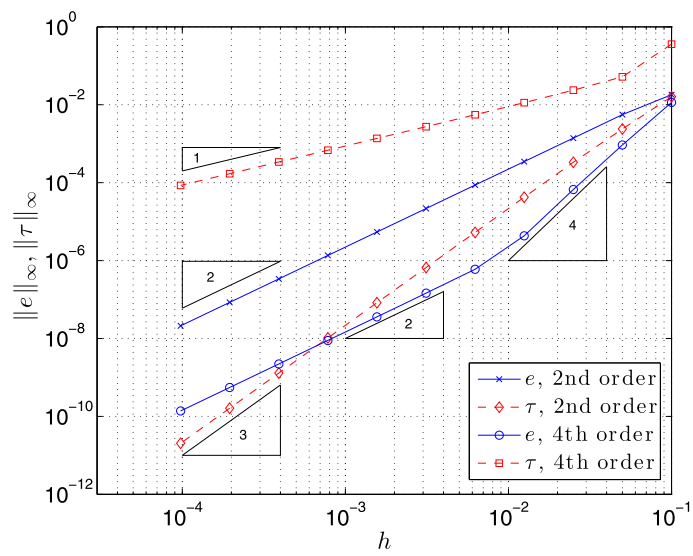

(b) Convergence of global error, second- and fourthorder scheme, $\delta_{0.9}$.

Fig. 7. Error behavior for non-uniform grid, $v=1 / 100$

$$
\frac{\mathrm{d} u}{\mathrm{~d} x}=\frac{\mathrm{d} u}{\mathrm{~d} \xi} / \frac{\mathrm{d} x}{\mathrm{~d} \xi},
$$

we observe that an $\mathcal{O}\left(h^{4}\right)$ approximation for both $\frac{\mathrm{d} u}{\mathrm{~d} \xi}$ and $\frac{\mathrm{d} x}{\mathrm{~d} \xi}$ is required. For example, Shishkin meshes (see e.g. [27,23]), which consist of two uniform meshes with a different mesh width, lack this smoothness and fourth order accuracy is not obtained, not even when the point of maximum error is in the interior. 


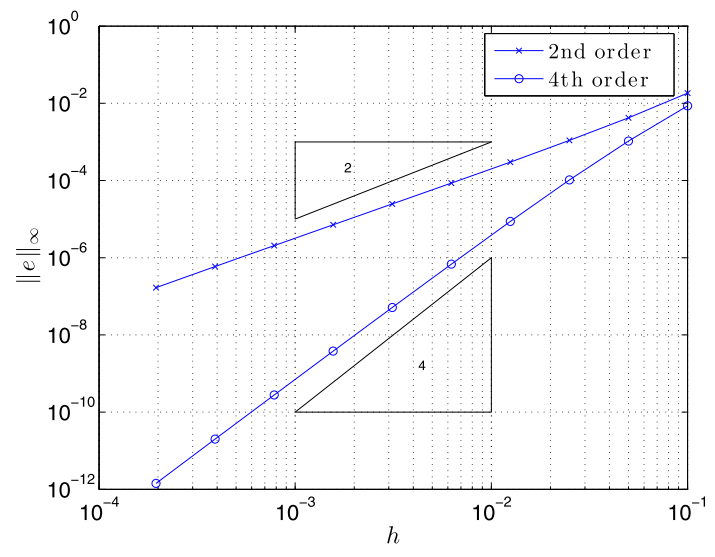

Fig. 8. Error behavior for non-uniform grid, $s=10 / h$.

\subsection{2 lid-driven cavity}

We continue the study of the order behavior of the fourth-order scheme by studying the steady lid-driven cavity flow at a Reynolds number of 1000 . In this benchmark problem [2] for the incompressible Navier-Stokes equations (NS) we introduce two major differences with the 1D convection-diffusion equation: (i) NS are non-linear, (ii) NS include a pressure term to satisfy the incompressibility constraint. To obtain steady solutions the full non-linear system of equations is solved with Newton linearization of the convective term. The resulting saddle-point matrix problem is solved with a direct solver. The iterative procedure is stopped when the residual (in the maximum norm) drops below $10^{-8}$, which requires approximately 6 iterations.

Similar to the one-dimensional boundary layer study, we have investigated both uniform and non-uniform exponential grids. The exponential grids are given by (161), with a stretching factor equal to the effective stretching factor of the cosine grid used by [2]:

$$
s=\frac{\frac{1}{2} \pi h}{\frac{1}{4} \pi^{2} h^{2}}=\frac{2}{\pi h} .
$$

The number of grid points ranges from $8 \times 8$ to $128 \times 128$.

Figs. 9 and 10 show some examples of solution profiles through the centerlines of the cavity, compared to the benchmark data of Botella and Peyret [2] (in the figures abbreviated as BP). The pressure is determined up to a constant, which has been chosen such that $p=0$ in the center of the cavity. It can be seen that the pressure converges to the correct values at the boundary, and that the boundary condition for the pressure is certainly not $\partial p / \partial n=0$. Note that the boundary conditions used by VV lead to a (numerical) boundary layer in the pressure, as shown in Figs. 11(a)-11(b). The velocity profiles along the centerlines are used to perform a quantitative comparison of the second and fourth order discretization on both the uniform and cosine grid. In order to measure the order of accuracy we compare the minimum value of $u$ along $x=0.5$ and maximum and minimum values of $v$ along $y=0.5$ with the benchmark data. The resulting error plots are shown in Fig. 12. The non-uniform grids are better able to capture the boundary layers and therefore lead to more accurate prediction of the minimum and maximum velocity values along the centerline. Comparing the second and fourth order results on the uniform grid, Fig. 12(a), we conclude that the fourth order method has a somewhat smaller error than the second order method, but converges only with second order upon grid refinement. This is in agreement with the error analysis from section 4 and the 1D results from section 5.1 - in particular Fig. 6(d).

On non-uniform grids we expect better convergence properties since the exponential grid satisfies $h_{1}=\mathcal{O}\left(h^{2}\right)$. Indeed, fourth order convergence of $u_{\max }$ and $v_{\max }$ is shown in Fig. 12(b). The convergence order of $v_{\min }$ is apparently lower; this can possibly be improved by adapting (165). Since this is problem specific we do not investigate this in detail here.

\subsection{Energy conservation in an inviscid cavity}

In this section we will show that the new boundary conditions for the fourth order method exactly conserve energy in case of no-penetration conditions. Desjardins et al. [3] considered a two-dimensional inviscid channel flow with periodic boundary conditions in one direction and no-slip conditions in the other. We increase the difficulty of their test case by prescribing non-zero tangential conditions on all boundaries (resulting in an inviscid cavity):

$$
\begin{array}{ll}
u(x, 0, t)=-1, & u(x, 1, t)=16 x^{2}(1-x)^{2}, \\
v(0, y, t)=1, & v(1, y, t)=-1 .
\end{array}
$$




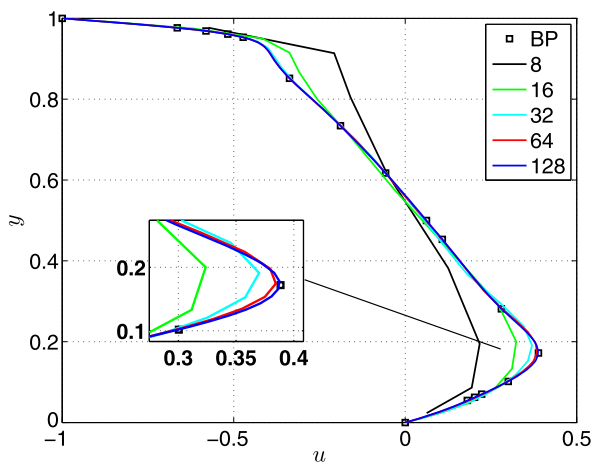

(a) 2nd order

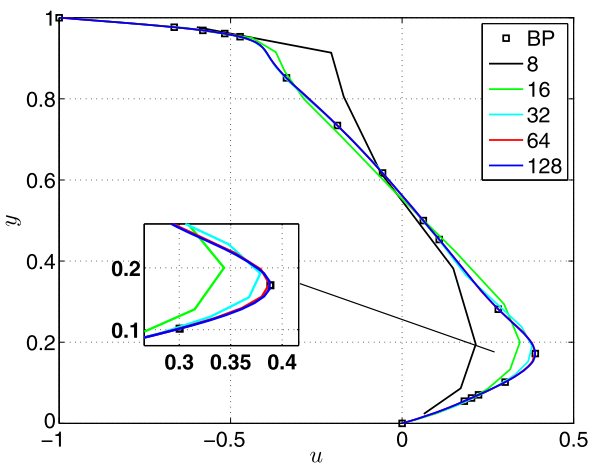

(b) 4 th order

Fig. 9. Velocity profiles $u(y)$ at $x=0.5,2$ nd and 4 th order method on an exponential grid.

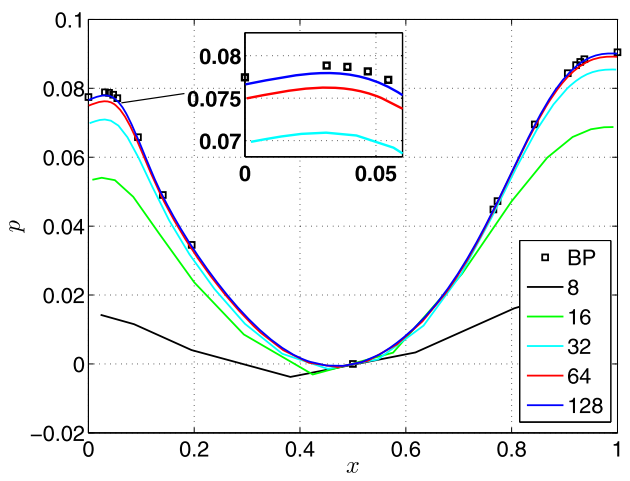

(a) 2nd order

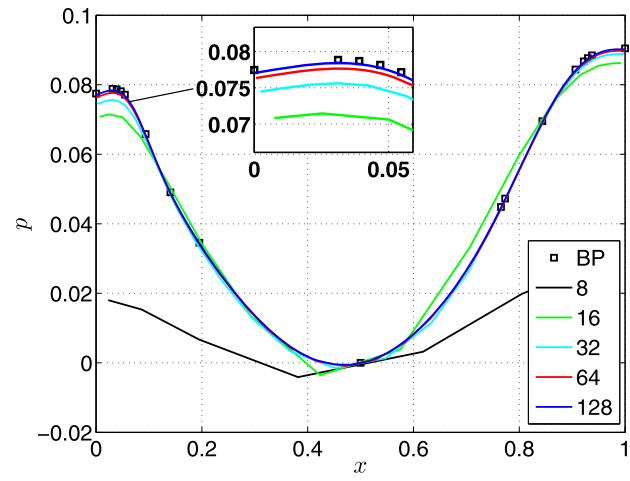

(b) 4 th order

Fig. 10. Pressure profiles $p(x)$ at $y=0.5,2$ nd and 4 th order method on an exponential grid.

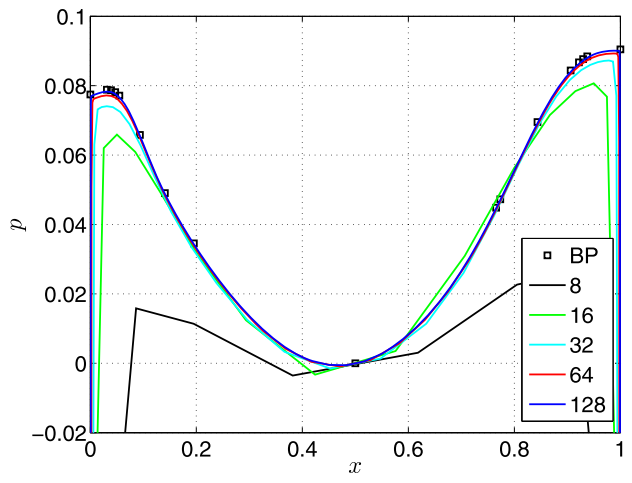

(a) Complete domain

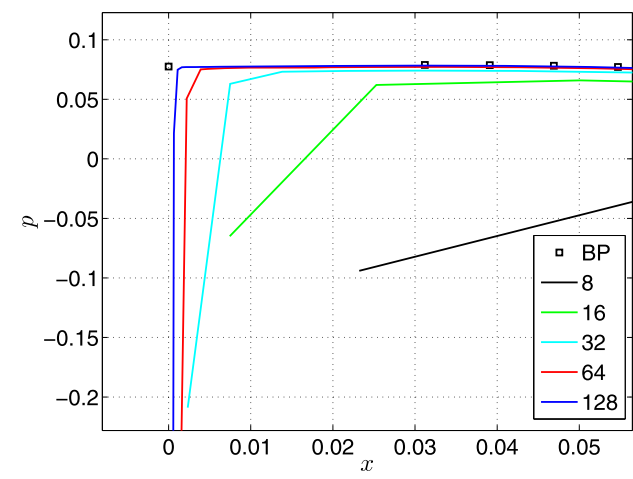

(b) Detail near $x=0$

Fig. 11. Pressure profiles $p(x)$ at $y=0.5$ with 4 th order method and old boundary conditions. Compare with Fig. 10.

See Fig. 13(a). The velocity field is discontinuous in the corners. Furthermore, in contrast to [3], we use the implicit midpoint method for time integration instead of the Crank-Nicolson method, since the latter is not truly energy-conserving [18].

The non-uniform grid that we have used consists of $20 \times 20$ volumes and is shown in Fig. 13(b). In $x$-direction the stretch factor (ratio between largest and smallest volume) is 2 , in $y$-direction it is 10 . The initial velocity field consists of uniform random numbers between -1 and 1 and is made divergence-free by a projection step involving the solution of a Poisson equation. This velocity field is subsequently advanced in time from $t=0$ to $t=1$ with $\Delta t=1 / 100$. In Fig. 14 we show the relative error in kinetic energy,

$$
e_{k}=\frac{k(t)-k(t=0)}{k(t=0)},
$$




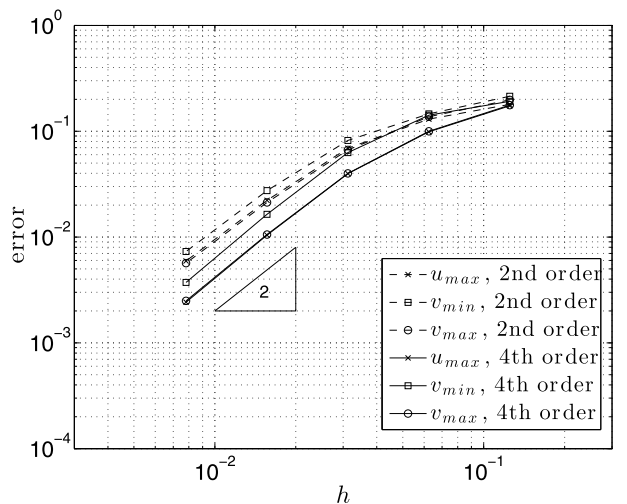

(a) Uniform grid

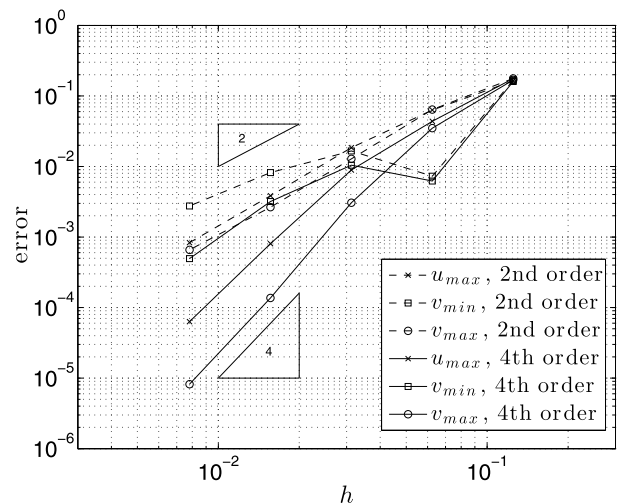

(b) Exponential grid

Fig. 12. Errors in velocity profiles.

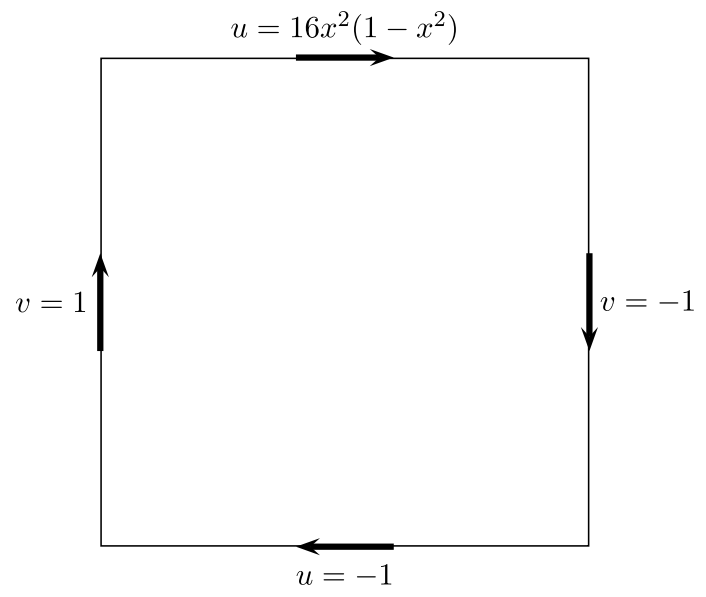

(a) Boundary conditions

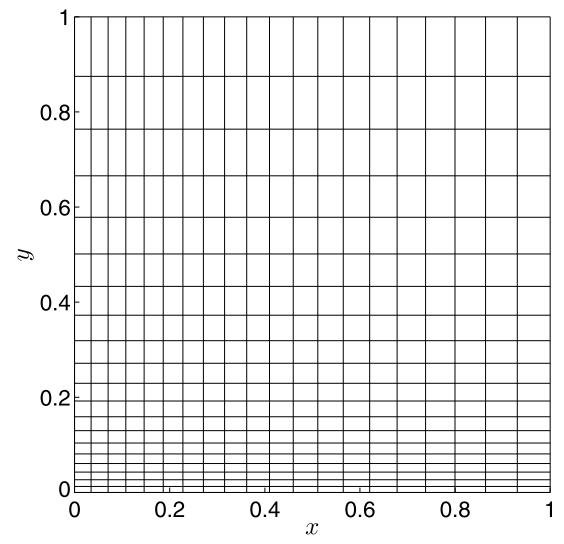

(b) Mesh

Fig. 13. Inviscid cavity

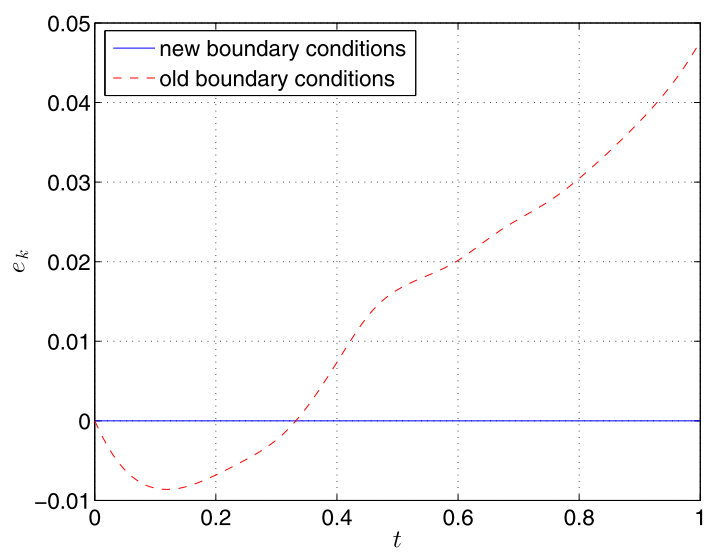

Fig. 14. Energy evolution in inviscid cavity as a function of time.

for both the new and old boundary condition treatment. With the new boundary conditions the energy error stays at machine precision $\left(\mathcal{O}\left(10^{-14}\right)\right)$, whereas the energy grows in time with the old boundary conditions. 


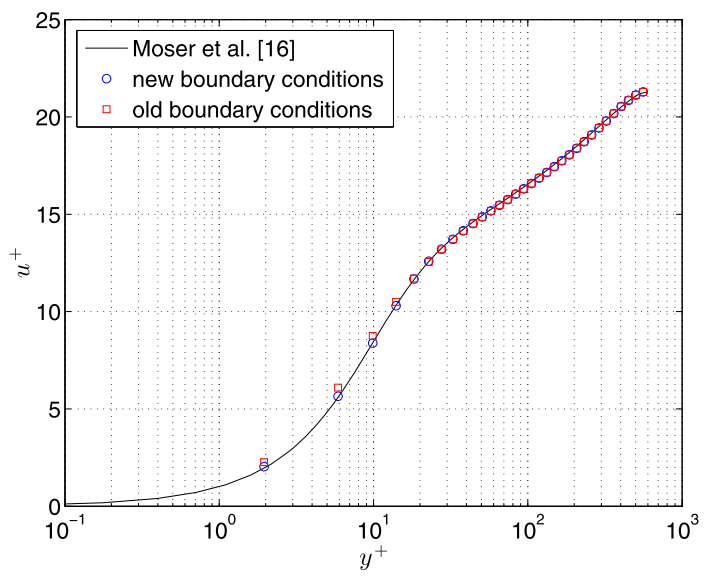

Fig. 15. Comparison of the mean streamwise velocity $u^{+}$as a function of $y^{+}$.

\subsection{Turbulent channel flow}

In this section, the two discrete boundary conditions given by (110) and (111) are compared for a turbulent channel flow. This flow can be seen as a prototype for near-wall turbulence. The Reynolds number in our simulation is based on the channel width and the bulk velocity. It is set equal to $R e=22,000$; in terms of the channel half width and the friction velocity the Reynolds number becomes approximately $R e_{\tau}=590$. Moser et al. [16] have performed a direct numerical simulation (DNS) of this channel flow. Their DNS data is often used to evaluate the accuracy of large eddy simulations (LES) of near-wall turbulence. The DNS was done on a $384 \times 257 \times 384$ grid; large eddy simulations typically use about $64^{3}$ points, i.e., they have about 144 times less points than the DNS of Moser et al. The present test considers an LES with a subgrid model that is based on the two non-zero invariants of the rate of strain tensor. Details of the subgrid model can be found in [24]. As usual the flow is assumed to be periodic in the stream- and spanwise direction. Consequently, the computational domain may be confined to a channel unit. The dimension of the considered unit is taken identical to that of the DNS. The LES uses a staggered grid with 64 uniformly distributed streamwise points and 32 (uniformly distributed) spanwise points. In the lower half of the channel, the wall-normal grid points are computed according to

$$
y_{j}=\frac{\sinh \left(\gamma j / N_{y}\right)}{2 \sinh (\gamma / 2)} \quad \text { with } j=0,1, \ldots, N_{y} / 2,
$$

where $N_{y}$ denotes the number of grid points in the wall-normal direction. The stretching parameter $\gamma$ is taken equal to 8 . The grid points in the upper half are computed by means of symmetry. The time step is set equal to $\Delta t=10^{-3}$ (non-dimensionalized by the bulk velocity and the channel width). Mean values of the velocity are computed by averaging the instantaneous velocity over the directions of periodicity, the two symmetrical halves of the channel, and over time. The averaging over time starts after a start-up period of 90,000 time steps. The averaging is performed over 90,000 time steps too. Fig. 15 shows a comparison of the two mean velocity profiles as obtained from our LES with the fourth-order symmetry-preserving discretization method, where we have applied the boundary treatment given by Eqs. (111) as well as that given by Eqs. (110). The difference between the resulting wall shear stresses is about 10\%. Eqs. (111) result into $\tau_{w}=2.9 \cdot 10^{-3}$, which is in excellent agreement with the DNS of Moser et al., whereas Eqs. (110) lead to $\tau_{w}=3.2 \cdot 10^{-3}$, which is too high. All results shown in Fig. 15 are scaled with the help of $\tau_{w}=2.9 \cdot 10^{-3}$. As can be seen, the main differences between the two wall treatments are located in the region near the wall. The differences are very small away from the wall (for $y^{+}>20$ ), whereas near the wall substantial differences, up to $10 \%$, can be observed.

Fig. 16 illustrates the differences between the resulting pressures. Away from the boundary $\left(y^{+}=0\right)$ the two pressures profiles differ by a constant (approximately). Such a difference by a constant is not relevant, since the pressure gradients are (almost) the same. Near the boundary $\left(0<y^{+}<20\right)$, on the other hand, the pressure profiles are different in the first few (4) grid points counted from the wall. The old boundary treatment described by Eqs. (110) results in a thin - numerical - boundary layer: the pressure is solved from a Poisson equation that is effectively supplied with Dirichlet conditions. The new boundary treatment, which effectively imposes a Neumann condition, leads to the proper boundary behavior of the pressure near the wall.

In conclusion, the boundary treatment given by (111) yields better results for both velocity and pressure in the boundary layer of a turbulent channel flow.

\section{Conclusions}

Symmetry-preserving or energy-conserving discretization methods have become popular methods for simulating turbulent flows governed by the incompressible Navier-Stokes equations. In this paper we have proposed a new consistent 


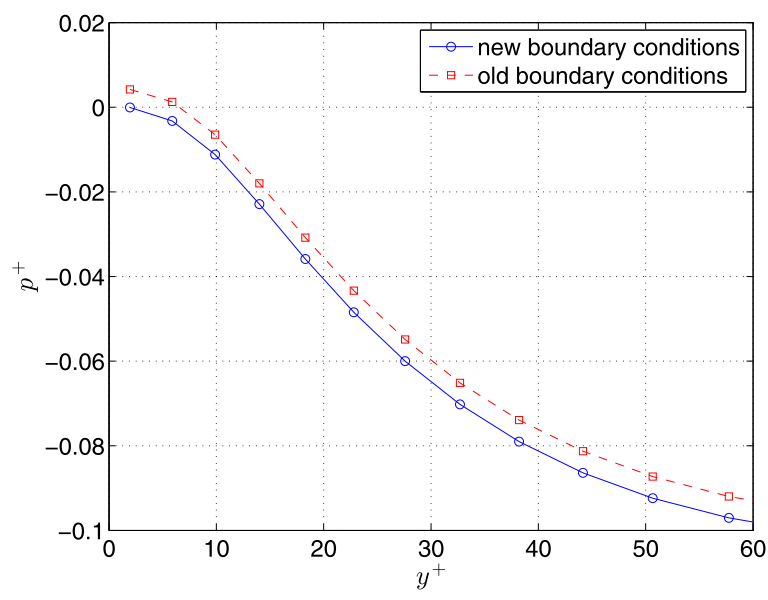

(a) Mean pressure $p^{+}$as a function of $y^{+}$

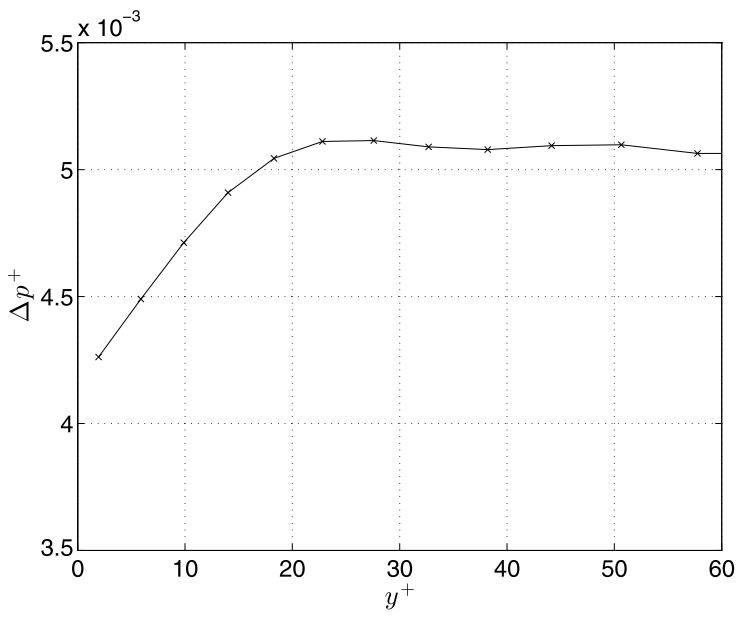

(b) The difference of the two pressures shown in figure 16a

Fig. 16. Comparison of pressure profiles.

boundary treatment for the symmetry-preserving discretization method described in [26]. The new boundary conditions are such that continuous integral identities (expressing conservation of mass, momentum and kinetic energy) are mimicked in a discrete sense, including the contributions from the boundaries. To derive this boundary treatment, we have used a new technique in which the 'implied' discretization of boundary volumes is derived based on global conservation of momentum. Upon requiring conservation of energy it turns out that the ghost values of the normal velocity component at a boundary should be set according to a usual Dirichlet condition, whereas the ghost values of the tangential velocity component at a boundary should be set according to a Neumann type condition. With these boundary conditions the convective operator is also energy-conserving if non-zero tangential boundary conditions are prescribed. The newly derived boundary conditions furthermore imply the correct boundary conditions for the pressure. The boundary conditions for the diffusive terms are unaltered and are of Dirichlet type.

The use of these conditions limits the global order of accuracy of the fourth order discretization method to second order on uniform grids, because the requirements which (skew-)symmetry pose on the coefficients of the matrices are not compatible with the requirements resulting from Taylor expansions to reach higher order accuracy. Here we have shown that by using properly refined non-uniform grids near the boundary, high-order accuracy of the global error can still be obtained. Since it is natural to apply grid refinement near solid boundaries, this forms no serious limitation. The simulation of a two-dimensional lid-driven cavity flow and of a three-dimensional turbulent channel flow confirmed the theoretical analysis and demonstrated the improvement with respect to previous boundary treatments.

\section{Appendix A. Interior discretization}

\section{A.1. Second order}

The divergence operator is given by

$$
(M u)_{i, j}=\left(M_{1} u\right)_{i, j}=\bar{u}_{i, j}-\bar{u}_{i-1, j}+\bar{v}_{i, j}-\bar{v}_{i, j-1} .
$$

The convective discretization for the $u$-component is given by

$$
\begin{aligned}
\left(C^{u}(\bar{u}, \bar{v}) u\right)_{i, j} & =\left(C_{1}^{u}(\bar{u}, \bar{v}) u\right)_{i, j} \\
& =\bar{u}_{i+1 / 2, j} u_{i+1 / 2, j}-\bar{u}_{i-1 / 2, j} u_{i-1 / 2, j}+\bar{v}_{i+1 / 2, j} u_{i, j+1 / 2}-\bar{v}_{i+1 / 2, j-1} u_{i, j-1 / 2}
\end{aligned}
$$

The convected $(u, v)$ and convecting velocities $(\bar{u}, \bar{v})$ are obtained by second order, grid-independent, interpolation of the form

$$
\bar{u}_{i+1 / 2, j}=\frac{1}{2}\left(\bar{u}_{i, j}+\bar{u}_{i+1, j}\right) .
$$

The pressure gradient discretization is given by

$$
\left(G^{u} p\right)_{i, j}=\left(G_{1}^{u} p\right)_{i, j}=\bar{p}_{i+1, j}-\bar{p}_{i, j}
$$

The diffusive terms are discretized as 


$$
\left(D^{u} u\right)_{i, j}=\left(D_{1}^{u} u\right)_{i, j}=\left(\overline{\frac{\partial u}{\partial x}}\right)_{i+1 / 2, j}-\left(\overline{\frac{\partial u}{\partial x}}\right)_{i-1 / 2, j}+\left(\overline{\frac{\partial u}{\partial y}}\right)_{i, j+1 / 2}-\left(\frac{\overline{\partial u}}{\partial y}\right)_{i, j-1 / 2},
$$

where

$$
\left(\frac{\overline{\partial u}}{\partial x}\right)_{i+1 / 2, j}=\frac{\bar{u}_{i+1, j}-\bar{u}_{i, j}}{\Delta x} .
$$

The diffusive discretization is the product of two first order operators and can be written in short as

$$
D_{1}^{u}=\Delta_{1}^{u} \Lambda_{1}^{u}\left(\Delta_{1}^{u}\right)^{T}
$$

where $\Delta_{1}^{u}$ is a simple differencing matrix consisting of $-1,0$ and 1 , and $\Lambda_{1}^{u}$ a diagonal matrix containing metric information $(\Delta y / \Delta x)$.

\section{A.2. Fourth order}

The divergence operator reads

$$
(M u)_{i, j}=\alpha_{1}\left(M_{1} u\right)_{i, j}-\left(M_{3} u\right)_{i, j}
$$

with

$$
\left(M_{3} u\right)_{i, j}=\overline{\bar{u}}_{i+1, j}-\overline{\bar{u}}_{i-2, j}+\overline{\bar{v}}_{i, j+1}-\overline{\bar{v}}_{i, j-2} \text {. }
$$

The convective discretization for the $u$-component reads

$$
C^{u}(\bar{u}, \bar{v}) u=\alpha_{1} C_{1}^{u}(\bar{u}, \bar{v}) u-C_{3}^{u}(\overline{\bar{u}}, \overline{\bar{v}}) u,
$$

where

$$
\left(C_{3}^{u}(\overline{\bar{u}}, \overline{\bar{v}}) u\right)_{i, j}=\overline{\bar{u}}_{i+3 / 2, j} u_{i+3 / 2, j}-\overline{\bar{u}}_{i-3 / 2, j} u_{i-3 / 2, j}+\overline{\bar{v}}_{i+1 / 2, j+1} u_{i, j+3 / 2}-\overline{\bar{v}}_{i+1 / 2, j-2} u_{i, j-3 / 2} .
$$

Like the divergence operator, the convective operator is a combination of two second order operators, with the exception that all convecting velocities $(\bar{u}, \bar{v}, \overline{\bar{u}}$ and $\overline{\bar{v}})$ are obtained by fourth order, grid-independent interpolation, e.g.:

$$
\overline{\bar{u}}_{i+3 / 2, j}=\frac{1}{2}\left(\beta_{1} \overline{\bar{u}}_{i, j}+\beta_{2} \overline{\bar{u}}_{i+1, j}+\beta_{2} \overline{\bar{u}}_{i+2, j}+\beta_{1} \overline{\bar{u}}_{i+3, j}\right)
$$

with $\beta_{1}=-\frac{1}{8}$ and $\beta_{2}=\frac{9}{8}$. The use of grid-independent interpolation is the critical difference between the method of Morinishi et al. [14] and the method of Verstappen and Veldman [26], the latter being employed here. The convected velocities are obtained as

$$
u_{i+3 / 2, j}=\frac{1}{2}\left(u_{i, j}+u_{i+3, j}\right) \text {. }
$$

For higher order (e.g. sixth order) the same approach should be followed [14]: an $n$th order combination of second order convective discretizations (like (A.11)), where each convective discretization contains an $n$th order convecting velocity (like (A.13)). The pressure gradient terms are, like the divergence operator, a linear combination of two second order operators:

$$
G^{u} p=\alpha_{1} G_{1}^{u} p-G_{3}^{u} p
$$

with

$$
\left(G_{3}^{u} p\right)_{i, j}=\overline{\bar{p}}_{i+3, j}-\overline{\bar{p}}_{i, j}
$$

The diffusive operator is obtained as the product of two fourth order operators, which ensures that the resulting operator is symmetric and positive definite. We focus on the fluxes in $x$-direction:

$$
\left(D^{u} u\right)_{i, j}=\left(\left(\alpha_{1} D_{1}^{u}-D_{3}^{u}\right) u\right)_{i, j}=\alpha_{1}\left(\left(\frac{\overline{\partial u}}{\partial x}\right)_{i+1 / 2, j}-\left(\frac{\overline{\partial u}}{\partial x}\right)_{i-1 / 2, j}\right)-\left(\left(\frac{\overline{\overline{\partial u}}}{\partial x}\right)_{i+3 / 2, j}-\left(\frac{\overline{\overline{\partial u}}}{\partial x}\right)_{i-3 / 2, j}\right) .
$$

As was the case for the convective operator, the fluxes on both the small and large volume are approximated to fourth order:

$$
\left(\frac{\overline{\partial u}}{\partial x}\right)_{i+1 / 2, j}=\frac{1}{72 \Delta x}\left(\alpha_{1}\left(\bar{u}_{i+1, j}-\bar{u}_{i, j}\right)-\left(\overline{\bar{u}}_{i+2, j}-\overline{\bar{u}}_{i-1, j}\right)\right)
$$

and similarly for $\left(\frac{\overline{\overline{\partial u}}}{\partial x}\right)_{i+3 / 2, j}$. In short, the diffusive discretization reads

$$
D^{u}=\left(\alpha_{1} \Delta_{1}^{u}-\Delta_{3}^{u}\right)\left(\alpha_{1} \Lambda_{1}^{u}-\Lambda_{3}^{u}\right)\left(\alpha_{1} \Delta_{1}^{u}-\Delta_{3}^{u}\right)^{T} .
$$




\section{Appendix B. Boundary contributions to the kinetic energy, fourth order method}

\section{B.1. Convective terms}

The convective matrix $C_{3}^{u}(\overline{\bar{u}}, \overline{\bar{v}})$ can be constructed once $u_{-1 / 2, j}$ and $u_{1 / 2, j}$ are expressed in terms of $u_{b}$ and interior points:

$$
\begin{gathered}
u_{-1 / 2, j}=\frac{1}{2}\left(u_{-2, j}+u_{1, j}\right)=u_{b}+\frac{1}{2} u_{1, j}-\frac{1}{2} u_{2, j}, \\
u_{1 / 2, j}=\frac{1}{2}\left(u_{-1, j}+u_{2, j}\right)=u_{b}-\frac{1}{2} u_{1, j}+\frac{1}{2} u_{2, j} .
\end{gathered}
$$

In Section 3.5 we comment on the use of higher-order interpolation formulas to obtain $u_{-1 / 2, j}$ and $u_{1 / 2, j}$. With these interpolations $C_{3}^{u}(\overline{\bar{u}}, \overline{\bar{v}})$ reads

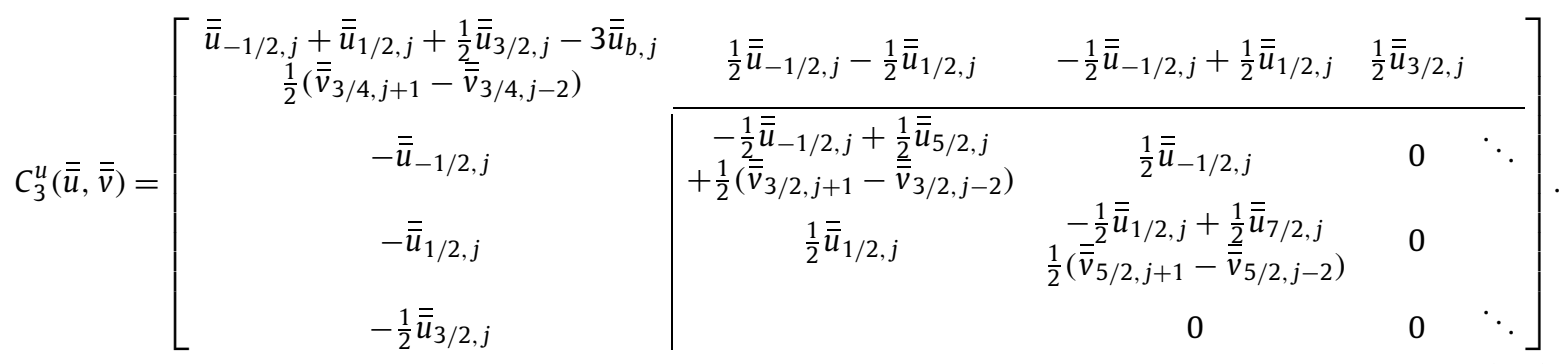

The rows represent the discretizations for the finite volumes around $u_{b}, u_{1}, u_{2}$, etc., whereas the columns represent the contribution from $u_{b}, u_{1}, u_{2}$, etc. The lower right part of the matrix is the interior discretization which is in practice used for solving the system of equations. Conservation of momentum as derived in Section 3.3 can be checked from the column sums of the matrix. We apply interpolation (A.13) and boundary conditions (35) and (40) and rewrite $C_{3}^{u}$. This is straightforward, except for the upper left entry of the matrix:

$$
\begin{aligned}
& \overline{\bar{u}}_{-1 / 2, j}+\overline{\bar{u}}_{1 / 2, j}+\frac{1}{2} \overline{\bar{u}}_{3 / 2, j}-3 \overline{\bar{u}}_{b, j}+\frac{1}{2}\left(\overline{\bar{v}}_{3 / 4, j+1}-\overline{\bar{v}}_{3 / 4, j-2}\right) \\
& =-\frac{1}{2} \overline{\bar{u}}_{b}+\frac{1}{2}\left(\overline{\bar{u}}_{3 / 2, j}-\overline{\bar{u}}_{b, j}+\overline{\bar{v}}_{3 / 4, j+1}-\overline{\bar{v}}_{3 / 4, j-2}\right) \\
& =-\frac{1}{2} \overline{\bar{u}}_{b}+\frac{1}{4}\left(\beta_{1} \overline{\bar{u}}_{b}+\beta_{2} \overline{\bar{u}}_{1}+\beta_{2} \overline{\bar{u}}_{2}+\beta_{1} \overline{\bar{u}}_{3}\right)-\frac{1}{2}\left(\beta_{1}+\beta_{2}\right) \overline{\bar{u}}_{b}+\frac{1}{2}\left(\overline{\bar{v}}_{3 / 4, j+1}-\overline{\bar{v}}_{3 / 4, j-2}\right) \\
& =-\frac{1}{2} \overline{\bar{u}}_{b}+\frac{1}{4}\left(\beta_{1}\left(\overline{\bar{u}}_{3, j}-\overline{\bar{u}}_{b, j}+\overline{\bar{v}}_{2, j+1}-\overline{\bar{v}}_{2, j-2}\right)+\beta_{2}\left(\overline{\bar{u}}_{2, j}+\overline{\bar{u}}_{1, j}-2 \overline{\bar{u}}_{b, j}+\overline{\bar{v}}_{1, j+1}-\overline{\bar{v}}_{1, j-2}\right)\right) \\
& =-\frac{1}{2} \overline{\bar{u}}_{b} .
\end{aligned}
$$

To arrive from Eq. (B.5) at Eq. (B.6) it is required to interpolate the vertical fluxes near the boundary as

$$
\overline{\bar{v}}_{3 / 4, j+1}=\frac{1}{2} \beta_{1} \overline{\bar{v}}_{2, j+1}+\frac{1}{2} \beta_{2} \overline{\bar{v}}_{1, j+1} \text {. }
$$

This leads to

$$
C_{3}^{u}(\overline{\bar{u}}, \overline{\bar{v}})=\left[\begin{array}{ccccc}
-\frac{1}{2} \overline{\bar{u}}_{b, j} & \overline{\bar{u}}_{b, j}-\overline{\bar{u}}_{1 / 2, j}-\overline{\bar{u}}_{b, j}+\overline{\bar{u}}_{1 / 2, j} & \frac{1}{2} \overline{\bar{u}}_{3 / 2, j} & \\
\cline { 2 - 5 } & 0 & \overline{\bar{u}}_{b, j}-\frac{1}{2} \overline{\bar{u}}_{1 / 2, j} & 0 & \ddots \\
-2 \overline{\bar{u}}_{b, j}+\overline{\bar{u}}_{1 / 2, j} & 0 & 0 & \\
-\overline{\bar{u}}_{1 / 2, j} & \frac{1}{2} \overline{\bar{u}}_{1 / 2, j} & 0 & \ddots
\end{array}\right] .
$$

In case $\overline{\bar{u}}_{b, j}=0$ the matrix is completely skew-symmetric, and $u^{T} C_{3}^{u} u=0$ : the discretization mimics exactly the property that the convective terms do not contribute to the global energy balance. For $\overline{\bar{u}}_{b, j} \neq 0$ we obtain

$$
u^{T} C_{3}^{u} u=-\frac{1}{2} \overline{\bar{u}}_{b, j}\left(u_{b, j}^{2}+2 u_{b, j}\left(u_{1, j}+u_{2, j}\right)-2 u_{1, j} u_{2, j}\right) .
$$

Taylor expanding $u_{1, j}$ and $u_{2, j}$ around $u_{b, j}$ gives 


$$
u^{T} C_{3}^{u} u=-\frac{3}{2} \overline{\bar{u}}_{b, j} u_{b, j}^{2}+\mathcal{O}\left(h^{2}\right)
$$

A similar analysis can be carried out for the $v$-component near a vertical boundary. The discretization of the volume surrounding $i=2$ requires an expression for $v_{0, j}$, since $v_{3 / 2, j}=\frac{1}{2}\left(v_{0, j}+v_{3, j}\right)$. We write the general expression

$$
v_{3 / 2, j}=\delta_{0} v_{b, j}+\delta_{1} v_{1, j}+\delta_{2} v_{2, j}+\delta_{3} v_{3, j},
$$

and determine the coefficients $\delta_{i}$ based on symmetry requirements. From this the boundary condition for $v_{0, j}$ can be derived. The convective matrix becomes

$$
C_{3}^{v}(\overline{\bar{u}}, \overline{\bar{v}})=\left[\begin{array}{c|cccc}
-2 \overline{\bar{u}}_{b, j+1 / 2}+\delta_{0} \overline{\bar{u}}_{1, j} & \delta_{1} \overline{\bar{u}}_{1, j+1 / 2}+\frac{1}{2} \overline{\bar{u}}_{2, j+1 / 2} & \delta_{2} \overline{\bar{u}}_{1, j+1 / 2} & \delta_{3} \overline{\bar{u}}_{1, j+1 / 2} & \frac{1}{2} \overline{\bar{u}}_{2, j+1 / 2} \\
-\overline{\bar{u}}_{b, j+1 / 2} & 0 & \frac{1}{2}\left(\overline{\bar{v}}_{1, j+3 / 2}-\overline{\bar{v}}_{1, j-3 / 2}\right) & \frac{1}{2} \overline{\bar{u}}_{3, j+1 / 2} & \\
-\delta_{0} \overline{\bar{u}}_{1, j+1 / 2} & 0 & \left.\overline{\bar{v}}_{2, j-3 / 2}\right) & 0 & \\
0 & -\delta_{1} \overline{\bar{u}}_{1, j+1 / 2} & -\delta_{2} \overline{\bar{u}}_{1, j+1 / 2} & \frac{1}{2} \overline{\bar{u}}_{4, j+1 / 2}-\delta_{3} \overline{\bar{u}}_{1, j+1 / 2} & \\
\left.\frac{1}{2} \overline{\bar{v}}_{2, j+3 / 2}-\overline{\bar{v}}_{2, j-3 / 2}\right) & \\
-\frac{1}{2} \overline{\bar{u}}_{2, j+1 / 2} & 0 & 0 &
\end{array}\right] .
$$

The rows represent the discretizations for the finite volumes around $v_{1}$, $v_{2}$, etc., whereas the columns represent the contribution from $v_{b}, v_{1}, v_{2}$, etc. Requiring that the convective discretization does not contribute to the kinetic energy in case $\overline{\bar{u}}_{b}=0$ gives the following solution for the coefficients: $\delta_{1}=\delta_{3}=\frac{1}{2}, \delta_{0}=\delta_{2}=0$. This means that the interpolation for $v_{3 / 2, j}$ reads

$$
v_{3 / 2, j}=\frac{1}{2}\left(v_{1, j}+v_{3, j}\right),
$$

and the implied boundary condition is

$$
v_{0, j}=v_{1, j} .
$$

So, like the fluxes $\overline{\bar{v}}$ and $\bar{v}$ (Eqs. (42)-(43)), the ghost value $v_{0, j}$ is obtained from a Neumann-type boundary condition. Rewriting the convective matrix using the divergence-free condition leads to

$$
C_{3}^{v}(\overline{\bar{u}}, \overline{\bar{v}})=\left[\begin{array}{c|ccccc}
-2 \overline{\bar{u}}_{b, j+1 / 2} & \overline{\bar{u}}_{b, j+1 / 2} & 0 & \frac{1}{2} \overline{\bar{u}}_{1, j+1 / 2} & \frac{1}{2} \overline{\bar{u}}_{2, j+1 / 2} \\
-\overline{\bar{u}}_{b, j+1 / 2} & 0 & \frac{1}{2} \overline{\bar{u}}_{b, j+1 / 2} & & & \ddots \\
0 & -\frac{1}{2} \overline{\bar{u}}_{1, j+1 / 2} & & 0 & & \\
0 & -\frac{1}{2} \overline{\bar{u}}_{2, j+1 / 2} & & & \ddots &
\end{array}\right] .
$$

For $\overline{\bar{u}}_{b, j+1 / 2}=0$ the matrix is completely skew-symmetric, and $v^{T} C_{3}^{v} v=0$. For non-homogeneous boundary data the contribution to the energy equation is

$$
v^{T} C_{3}^{v} v=\overline{\bar{u}}_{b, j+1 / 2}\left(-2 v_{b, j} v_{1, j}-v_{b, j} v_{2, j}+v_{1, j}^{2}+\frac{1}{2} v_{2, j}^{2}\right) \text {. }
$$

Using Taylor expansions for $v_{1}$ and $v_{2}$ around $v_{b}$ then gives

$$
v^{T} C_{3}^{v} v=-\frac{3}{2} v_{b, j}^{2} \overline{\bar{u}}_{b, j+1 / 2}+\mathcal{O}\left(h^{2}\right) .
$$

\section{B.2. Diffusive terms}

The contribution of the diffusive terms to the energy equation reads

$$
\begin{aligned}
u^{T} D^{u} u= & u_{b, j}\left[\alpha_{1}\left(\left(\frac{\overline{\partial u}}{\partial x}\right)_{1 / 2, j}-\left(\frac{\overline{\partial u}}{\partial x}\right)_{b, j}\right)_{-}\left(\left(\frac{\overline{\bar{u}}}{\partial x}\right)_{-1 / 2, j}+\left(\frac{\overline{\overline{\partial u}}}{\partial x}\right)_{1 / 2, j}+\left(\frac{\overline{\overline{\partial u}}}{\partial x}\right)_{3 / 2, j}-3\left(\frac{\overline{\overline{\partial u}}}{\partial x}\right)_{b, j}\right)\right] \\
& +u_{1, j}\left[\alpha_{1}\left(\left(\frac{\overline{\partial u}}{\partial x}\right)_{3 / 2, j}-\left(\frac{\overline{\partial u}}{\partial x}\right)_{1 / 2, j}\right)-\left(\left(\frac{\overline{\bar{u}}}{\partial x}\right)_{5 / 2, j}-\left(\frac{\overline{\overline{\partial u}}}{\partial x}\right)_{-1 / 2, j}\right)\right] \\
& +u_{2, j}\left[\alpha_{1}\left(\left(\frac{\overline{\partial u}}{\partial x}\right)_{5 / 2, j}-\left(\frac{\overline{\partial u}}{\partial x}\right)_{3 / 2, j}\right)-\left(\left(\frac{\overline{\bar{u}}}{\partial x}\right)_{7 / 2, j}-\left(\frac{\overline{\overline{\partial u}}}{\partial x}\right)_{1 / 2, j}\right)\right]+\cdots
\end{aligned}
$$

Regrouping gives 


$$
\begin{aligned}
& -\left(\frac{\overline{\partial u}}{\partial x}\right)_{b, j}\left(\alpha_{1}-9\right) u_{b, j}+\left(\frac{\overline{\partial u}}{\partial x}\right)_{-1 / 2, j} 3\left(u_{1, j}-u_{b, j}\right)-\left(\frac{\overline{\partial u}}{\partial x}\right)_{1 / 2, j}\left(\alpha_{1}\left(u_{1, j}-u_{b, j}\right)-3\left(u_{2, j}-u_{b, j}\right)\right) \\
& -\left(\frac{\overline{\partial u}}{\partial x}\right)_{3 / 2, j}\left(\alpha_{1}\left(u_{2, j}-u_{1, j}\right)-3\left(u_{3, j}-u_{b, j}\right)\right)+\cdots .
\end{aligned}
$$

The first term is recognized as the discrete counterpart of the boundary integral in (69). The last term is handled like the interior terms. Since the first order derivatives are discretized as

$$
\left(\frac{\overline{\partial u}}{\partial x}\right)_{i+1 / 2, j}=\frac{\Delta y}{72 \Delta x}\left(\alpha_{1}\left(u_{i+1, j}-u_{i, j}\right)-3\left(u_{i+2, j}-u_{i-1, j}\right)\right),
$$

it can be written as

$$
-\left(\frac{\overline{\partial u}}{\partial x}\right)_{3 / 2, j}\left(\alpha_{1}\left(u_{2, j}-u_{1, j}\right)-3\left(u_{3, j}-u_{b, j}\right)\right)=-72 \Delta x \Delta y\left(\frac{9}{8} \frac{u_{2, j}-u_{1, j}}{\Delta x}-\frac{1}{8} \frac{u_{3, j}-u_{b, j}}{3 \Delta x}\right)^{2} .
$$

This is the discrete equivalent of $\int\left(\frac{\partial u}{\partial x}\right)^{2} \mathrm{~d} x$, centered around $(i+3 / 2, j)$. The second and third term in (B.20) should provide a similar approximation to a volume centered around $(i+1 / 2, j)$. Evaluating (B.21) for $i=0$ and $i=-1$ and 'standard' boundary conditions

$$
\begin{aligned}
& u_{-2, j}=2 u_{b, j}-u_{2, j}, \\
& u_{-1, j}=2 u_{b, j}-u_{1, j},
\end{aligned}
$$

gives

$$
\begin{aligned}
\left(\frac{\overline{\partial u}}{\partial x}\right)_{1 / 2, j} & =\frac{\Delta y}{72 \Delta x}\left(\alpha_{1}\left(u_{1, j}-u_{b, j}\right)-3\left(u_{2, j}+u_{1, j}-2 u_{b, j}\right)\right), \\
\left(\frac{\overline{\partial u}}{\partial x}\right)_{-1 / 2, j} & =\left(\frac{\overline{\partial u}}{\partial x}\right)_{1 / 2, j} .
\end{aligned}
$$

Adding the second and third term in (B.20) gives

$$
\begin{aligned}
& \left(\frac{\overline{\partial u}}{\partial x}\right)_{-1 / 2, j} 3\left(u_{1, j}-u_{b, j}\right)-\left(\frac{\overline{\partial u}}{\partial x}\right)_{1 / 2, j}\left(\alpha_{1}\left(u_{1, j}-u_{b, j}\right)-3\left(u_{2, j}-u_{b, j}\right)\right) \\
& =-\left(\frac{\overline{\partial u}}{\partial x}\right)_{1 / 2, j}\left(\alpha_{1}\left(u_{1, j}-u_{b, j}\right)-3\left(u_{2, j}+u_{1, j}-2 u_{b, j}\right)\right) \\
& =-72 \Delta x \Delta y\left(\frac{9}{8} \frac{u_{1, j}-u_{b, j}}{\Delta x}-\frac{1}{8} \frac{u_{2, j}+u_{1, j}-2 u_{b, j}}{3 \Delta x}\right)^{2} .
\end{aligned}
$$

Taking all steps together, Eq. (B.19) can be written as

$$
\begin{aligned}
u^{T} D^{u} u= & -\left(\frac{\overline{\partial u}}{\partial x}\right)_{b, j}\left(\alpha_{1}-9\right) u_{b, j}+\cdots \\
& -72 \Delta x \Delta y\left[\left(\frac{9}{8} \frac{u_{1, j}-u_{b, j}}{\Delta x}-\frac{1}{8} \frac{u_{2, j}+u_{1, j}-2 u_{b, j}}{3 \Delta x}\right)^{2}+\left(\frac{9}{8} \frac{u_{2, j}-u_{1, j}}{\Delta x}-\frac{1}{8} \frac{u_{3, j}-u_{b, j}}{3 \Delta x}\right)^{2}+\cdots\right] .
\end{aligned}
$$

This is the discrete equivalent of the right-hand side of (69).

For the $v$-component a similar analysis can be carried out.

\section{Appendix C. Boundary stencil for the Poisson equation}

In the interior the $u$-momentum equation reads:

$$
\dot{u}_{i, j}=N_{i, j}^{u}-\left(\Omega_{i, j}^{u}\right)^{-1}\left(G^{u} p\right)_{i, j},
$$

where $N^{u}$ includes the convective and diffusive terms of the $u$-component, divided by the effective finite volume size (see Eq. (30)). The time derivative of the divergence equation reads 


$$
\alpha_{1}\left(\dot{u}_{i, j}-\dot{u}_{i-1, j}\right)-\alpha_{2}\left(\dot{u}_{i+1, j}-\dot{u}_{i-2, j}\right)+(v \text {-component })=0 .
$$

Inserting (C.1) gives the Laplace operator for a uniform grid:

$$
\begin{aligned}
& \frac{1}{\Delta x}\left(\frac{1}{8} p_{i-3, j}-\frac{27}{4} p_{i-2, j}+\frac{783}{8} p_{i-1, j}-\frac{365}{2} p_{i, j}+\frac{783}{8} p_{i+1, j}-\frac{27}{4} p_{i+2, j}+\frac{1}{8} p_{i+3, j}\right) \\
& \quad=\alpha_{1}\left(N_{i, j}^{u}-N_{i-1, j}^{u}\right)-\alpha_{2}\left(N_{i+1, j}^{u}-N_{i-2, j}^{u}\right)+(v \text {-component }) .
\end{aligned}
$$

Near a boundary the stencil of the Laplace operator is found in a similar way. Taking the time derivative of the divergencefree constraint near the boundary (the first row of Eq. (36)) gives

$$
\left(\alpha_{1}-\alpha_{2}\right) \dot{u}_{1, j}-\alpha_{2} \dot{u}_{2, j}+\left(-\alpha_{1}+2 \alpha_{2}\right) \dot{u}_{b, j}=0,
$$

where we have ignored the $v$-component. Substituting the momentum equations for $\dot{u}_{1, j}$ and $\dot{u}_{2, j}$ gives

$$
\frac{1}{\Delta x}\left(-\frac{677}{8} p_{1 j}+\frac{729}{8} p_{2, j}-\frac{53}{8} p_{3, j}+\frac{1}{8} p_{4, j}\right)=\left(\alpha_{1}-\alpha_{2}\right) N_{1, j}^{u}-\alpha_{2} N_{2, j}^{u}+\left(-\alpha_{1}+2 \alpha_{2}\right) \dot{u}_{b, j} .
$$

The effective ('implied') boundary condition for the pressure follows by subtracting Eq. (C.3) (for $i=1$ ) from the last equation:

$$
\frac{1}{\Delta x}\left(\frac{1}{8}\left(p_{3, j}-p_{-2, j}\right)-\frac{27}{4}\left(p_{2, j}-p_{-1, j}\right)+\frac{783}{8}\left(p_{1, j}-p_{0, j}\right)\right)=\alpha_{1} N_{b, j}^{u}-\alpha_{2}\left(N_{-1, j}^{u}+N_{1, j}^{u}\right)+\left(-\alpha_{1}+2 \alpha_{2}\right) \dot{u}_{b, j} .
$$

Repeating this procedure for $i=2$ gives the following stencil for the Poisson operator:

$$
\frac{1}{\Delta x}\left(\frac{729}{8} p_{1, j}-\frac{1459}{8} p_{2, j}+\frac{783}{8} p_{3, j}-\frac{27}{4} p_{4, j}+\frac{1}{8} p_{5, j}\right)=\alpha_{1}\left(N_{2, j}^{u}-N_{1, j}^{u}\right)-\alpha_{2} N_{3, j}^{u}+\alpha_{2} \dot{u}_{b, j},
$$

so the effective boundary condition is

$$
\frac{1}{\Delta x}\left(\frac{1}{8}\left(p_{2, j}-p_{-1, j}\right)-\frac{27}{4}\left(p_{1, j}-p_{0, j}\right)\right)=-\alpha_{2} N_{b, j}^{u}+\alpha_{2} \dot{u}_{b, j} .
$$

Similarly, for $i=3$, the stencil of the Poisson operator is

$$
\frac{1}{\Delta x}\left(-\frac{53}{8} p_{1, j}+\frac{783}{8} p_{2, j}-\frac{365}{2} p_{3, j}+\frac{783}{8} p_{4, j}-\frac{27}{4} p_{5, j}+\frac{1}{8} p_{6, j}\right)=\alpha_{1}\left(N_{3, j}^{u}-N_{2, j}^{u}\right)-\alpha_{2}\left(N_{4, j}^{u}-N_{1, j}^{u}\right),
$$

and the effective boundary condition is

$$
\frac{1}{\Delta x} \frac{1}{8}\left(p_{1, j}-p_{0, j}\right)=0 \text {. }
$$

\section{References}

[1] O. Axelsson, L. Kolotilina, Monotonicity and discretization error estimates, SIAM J. Numer. Anal. 27 (6) (1990) 1591-1611.

[2] O. Botella, R. Peyret, Benchmark spectral results on the lid-driven cavity flow, Comput. Fluids 27 (4) (1998) 421-433.

[3] O. Desjardins, G. Blanquart, G. Balarac, H. Pitsch, High order conservative finite difference scheme for variable density low Mach number turbulent flows, J. Comput. Phys. 227 (2008) 7125-7159.

[4] P.M. Gresho, R.L. Sani, Incompressible Flow and the Finite Element Method. Vol. 2: Isothermal Laminar Flow, Wiley, 2000.

[5] F.E. Ham, F.S. Lien, A.B. Strong, A fully conservative second-order finite difference scheme for incompressible flow on nonuniform grids, J. Comput. Phys. 177 (2002) 117-133.

[6] F.H. Harlow, J.E. Welch, Numerical calculation of time-dependent viscous incompressible flow of fluid with free surface, Phys. Fluids 8 (1965) 2182-2189.

[7] W. Hundsdorfer, J. Verwer, Numerical Solution of Time-Dependent Advection-Diffusion-Reaction Equations, Springer, 2007.

[8] J. Lorenz, Zur Inversmonotonie diskreter Probleme, Numer. Math. 27 (1977) 227-238.

[9] K. Mahesh, G. Constantinescu, P. Moin, A numerical method for large-eddy simulation in complex geometries, J. Comput. Phys. 197 (2004) $215-240$.

[10] T.A. Manteuffel, A.B. White, The numerical solution of second-order boundary value problems on non-uniform meshes, Math. Comput. 47 (176) (1986) 511-535.

[11] R.M.M. Mattheij, S.W. Rienstra, J.H.M. ten Thije Boonkkamp, Partial Differential Equations: Modeling, Analysis, Computation, SIAM, 2005.

[12] K. Mattson, J. Nordstrøm, Summation by parts operators for finite difference approximations of second derivatives, J. Comput. Phys. 199 (2004) 503-540.

[13] R. Mittal, P. Moin, Suitability of upwind-biased finite difference schemes for large-eddy simulation of turbulent flows, AIAA J. 35 (8) (1997).

[14] Y. Morinishi, T.S. Lund, O.V. Vasilyev, P. Moin, Fully conservative higher order finite difference schemes for incompressible flows, J. Comput. Phys. 143 (1998) 90-124.

[15] K.W. Morton, D.F. Mayers, Numerical Solution of Partial Differential Equations, Cambridge University Press, 2005.

[16] R.D. Moser, J. Kim, N.N. Mansour, Direct numerical simulation of turbulent channel flow up to $R e_{\tau}=590$, Phys. Fluids 11 (1999) $943-945$.

[17] B. Perot, Conservation properties of unstructured staggered mesh schemes, J. Comput. Phys. 159 (2000) 58-89. 
[18] B. Sanderse, Energy-conserving Runge-Kutta methods for the incompressible Navier-Stokes equations, J. Comput. Phys. 233 (2012) 100-131, http://dx.doi.org/10.1016/j.jcp.2012.07.039.

[19] R.L. Sani, J. Shen, O. Pironneau, P.M. Gresho, Pressure boundary condition for the time-dependent incompressible Navier-Stokes equations, Int. J. Numer. Methods Fluids 50 (2006) 673-682.

[20] B. Strand, Summation by parts for finite difference approximations for d/dx, J. Comput. Phys. 110 (1994) $47-67$.

[21] O.V. Vasilyev, High order finite difference schemes on non-uniform meshes with good conservation properties, J. Comput. Phys. 157 (2000) 746-761.

[22] A.E.P. Veldman, "Missing" boundary conditions? Discretize first, substitute next, and combine later, SIAM J. Sci. Stat. Comput. 11 (1990) $82-91$.

[23] A.E.P. Veldman, High-order symmetry-preserving discretization of convection-diffusion equations on strongly stretched grids, in: G. Lube, G. Rapin (Eds.), Proceedings of the Int. Conf. on Boundary and Interior Layers, 2006.

[24] R.W.C.P. Verstappen, When does eddy viscosity damp subfilter scales sufficiently?, J. Sci. Comput. 49 (2011) 94-110.

[25] R.W.C.P. Verstappen, A.E.P. Veldman, Direct numerical simulation of turbulence at lower costs, J. Eng. Math. 32 (1997) 143-159.

[26] R.W.C.P. Verstappen, A.E.P. Veldman, Symmetry-preserving discretization of turbulent flow, J. Comput. Phys. 187 (2003) $343-368$.

[27] P. Wesseling, Principles of Computational Fluid Dynamics, Springer, 2001. 